\title{
autoDIAS: A Python Tool for an Automated Distortion/Interaction Activation Strain Analysis
}

Dennis Svatunek, Kendall N. Houk

Submitted date: 20/05/2019 Posted date: 21/05/2019

Licence: CC BY-NC-ND 4.0

Citation information: Svatunek, Dennis; Houk, Kendall N. (2019): autoDIAS: A Python Tool for an Automated Distortion/Interaction Activation Strain Analysis. ChemRxiv. Preprint.

The Distortion/Interaction Activation Strain (DIAS) analysis is a powerful tool for the investigation of energy barriers. However, setup and data analysis of such a calculation can be cumbersome and requires lengthy intervention of the user. We present autoDIAS, a python tool for the automated setup, performance, and data extraction of the DIAS analysis, including automated detection of fragments and relevant geometric parameters.

File list (4)

autoDIAS.pdf (682.74 KiB)

autoDIAS.png (64.09 KiB)

SI.pdf (380.93 KiB)

autoDIAS-1.0.1.zip (22.28 KiB) view on ChemRxiv - download file

view on ChemRxiv - download file

view on ChemRxiv - download file

view on ChemRxiv - download file 


\title{
autoDIAS: a python tool for an automated Distortion/Interaction Activation Strain Analysis
}

\author{
Dennis Svatunek* and Kendall N. Houk \\ Correspondence to: Dennis Svatunek (E-mail: d.svatunek@chem.ucla.edu)
}

\begin{abstract}
The Distortion/Interaction Activation Strain (DIAS) analysis is a powerful tool for the investigation of energy barriers. However, setup and data analysis of such a calculation can be cumbersome and requires lengthy intervention of the user. We present autoDIAS, a python tool for the automated setup, performance, and data extraction of the DIAS analysis, including automated detection of fragments and relevant geometric parameters.
\end{abstract}

\section{Introduction}

The Distortion Interaction Analysis, ${ }^{1}$ also known as Activation Strain Model (ASM), ${ }^{2,3}$ is an energy decomposition method which allows for chemically intuitive analysis of energy barriers. ${ }^{4}$ In the Distortion/Interaction Activation Strain (DIAS) analysis, which is a further development of energy decompositions introduced by Morokuma, ${ }^{5}$ the electronic energy $\Delta E$ of a complex, for example a transition state geometry, is dissected into two contributing factors.

$\Delta E_{=} \Delta E_{\text {dist }}+\Delta E_{\text {int }}$

The distortion energy $\Delta \mathrm{E}_{\text {dist }}$ (called strain energy $\Delta \mathrm{E}_{\text {strain }}$ in the Activation Strain Model) describes the energy needed to distort the isolated fragments from their relaxed geometries to the geometry found in the complex. The interaction energy $\Delta \mathrm{E}_{\text {int }}$ describes the usually stabilizing interaction between the fragments if they are brought together into the complex. This analysis can be applied to single geometries or multiple geometries along a scan or reaction coordinate, to provide information on the nature of interaction between the fragments and the potential energy surface. This method has been widely applied for a broad range of chemical systems such as cycloadditions, ${ }^{6-18}$ ene reactions, ${ }^{19}$ metal complexes and catalysis, ${ }^{20-27}$ organocatalysis, ${ }^{28-31}$ and addition $^{32}$ and substitution reactions. ${ }^{33-35}$

The implementation of a DIAS analysis is straight forward and can be performed using any electronic structure software. It requires the calculation of the electronic energies of the relaxed fragments, the complex and the distorted fragments. The distortion energy for each fragment can then be calculated by subtracting the energy of the relaxed geometry while the interaction energy is the difference between the sum of distortion energies and the energy of the complex (Figure 1).
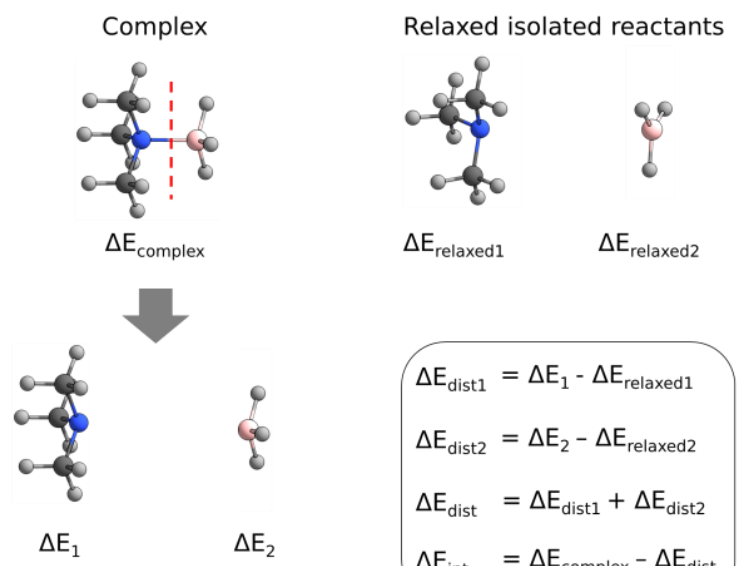

Distorted isolated reactants

Figure 1. Calculations performed during a Distortion/Interaction Activation Strain analysis.

While this is, in theory, simple to realize it requires a large amount of effort in the preparation of the input files for the separated distorted fragments and the analysis regarding geometric parameters and energies. In addition, it has been shown that performing a DIAS analysis along a reaction or scan coordinate, rather than on a single point like a transition state, can be beneficial. ${ }^{36,37}$ However, this 
requires the preparation and analysis of potentially large amounts of structures. Automation of these processes is needed for simple and efficient access to such DIAS analyses. To the best of our knowledge only few software packages aim to solve this problem; however, they are limited in functionality or to a certain electronic structure program. PyFrag, ${ }^{38}$ the python tool that inspired autoDIAS, is a wrapper for the Amsterdam Density Functional (ADF, SCM, the Netherlands $)^{39,40}$ software package. It allows for performance of a semi-automated Distortion/Interaction Activation Strain analysis, in this case called Activation Strain Model, and additional energy decomposition methods provided within ADF. While an excellent tool for the use with ADF it is limited to this electronic structure program. Recently Laloo et al. published an extension to the ExcelAutomat ${ }^{41}$ that aims for preparation of input files and plotting of the resulting data. ${ }^{42}$ While this makes good progress towards automation of the process, it does not provide a fully automated integration of a DIAS analysis and is limited in features.

We present autoDIAS, a python-based tool for the automatic setup, execution and data analysis of DIAS analyses. This tool aims for versatility and a highly automated workflow. It allows running the Distortion/Interaction Activation Strain analysis on single geometries or a series of geometries as in scan or IRC coordinates using any quantum chemical software packages (drivers) that accepts input of geometries in Cartesian coordinates, e.g. Gaussian, ORCA, NWChem, and Q-Chem. Basic data analysis was implemented for these four software packages ${ }^{43}$ but can easily be expanded. Geometry input can be provided through commonly used $X Y Z$ files or Gaussian output files. Relevant energies are calculated and geometric parameters for each structure, such as distances, angles, and dihedral angles, are extracted and composed into a single file for further handling like plotting of the calculated information. Figure 2 provides an overview of the workflow implemented in autoDIAS.

In addition, autoDIAS includes several useful utilities, features and modes, including a reduction of the amount of calculated points in multi geometry DIAS calculations, a reordering of structures from IRC calculations to present a continuous progress along the reaction coordinate, an automated detection of fragments, and an automated detection of forming/breaking bonds for multi geometry calculations.

\section{Examples}

In the following three examples of DIAS analyses are presented which were prepared using autoDIAS on the Hoffman2 cluster at UCLA. Gaussian 09 was used to prepare the input structures and as driver for the required single point energy calculations.

\section{Reaction 1}

The DIAS analysis was conducted on the transition state geometry of the cycloaddition between a tertiary azide and the strained cyclooctyne (1R,8S,9s)-Bicyclo[6.1.0]non-4-yn-9-ylmethanol (BCN) taken from a recent study about the reactivity of tertiary azides in strain-promoted alkyne-azide cycloadditions (Figure 3). ${ }^{7}$ Calculations were performed at the B3LYP/6-31G(d) level of theory. Fragments were automatically and correctly identified by autoDIAS. Input and structures were parsed in $35 \mathrm{~ms}$, the required three Gaussian input files were prepared in $18 \mathrm{~ms}$. The electronic energy of the transition state in relation to isolated reactants is $9.7 \mathrm{kcal} / \mathrm{mol} . \Delta \mathrm{E}_{\text {int }}$ is stabilizing with a value of $-6.7 \mathrm{kcal} / \mathrm{mol}$ and $\Delta \mathrm{E}_{\text {dist }}$ is destabilizing with a total value of $16.4 \mathrm{kcal} / \mathrm{mol}$. The tertiary azide contributes mainly to this distortion energy with $14.7 \mathrm{kcal} / \mathrm{mol}$ while the distortion energy for $\mathrm{BCN}$ is only $1.7 \mathrm{kcal} / \mathrm{mol}$.
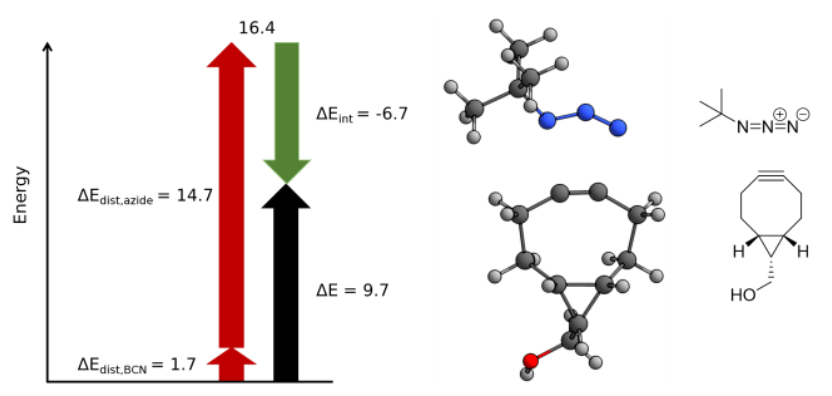

Figure 3. DIAS analysis of the transition state between BCN and tert-butylazide. Energies in $\mathrm{kcal} / \mathrm{mol}$. 
autoDIAS

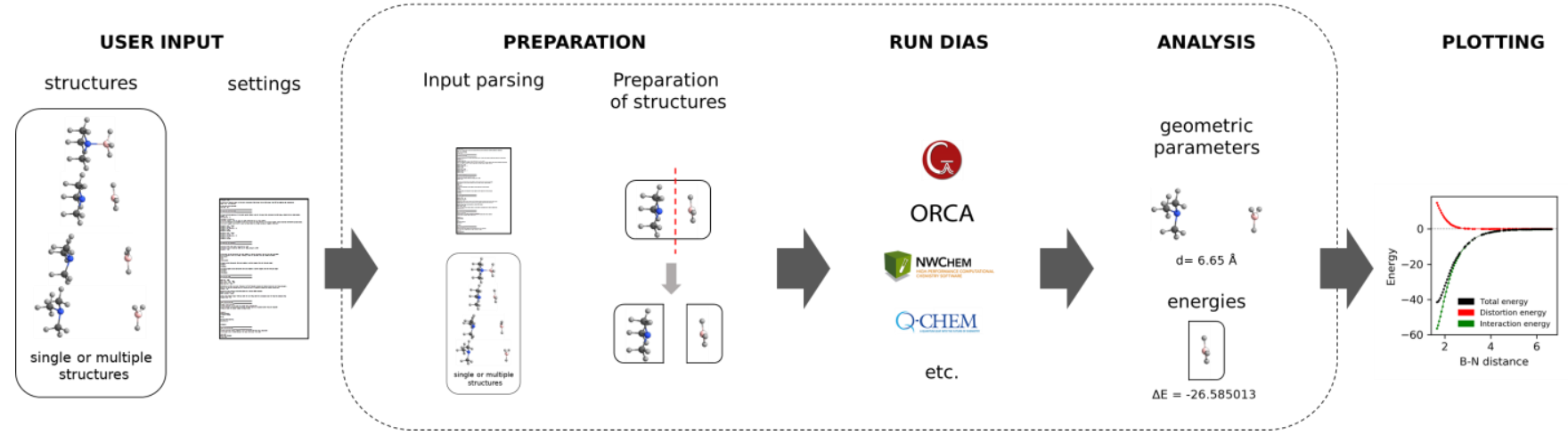

Figure 2. Workflow of autoDIAS.

\section{Scan 1}

A scan with 100 steps along the $B-N$ distance in the trimethylamine-borane complex from 1.65 to $3.65 \AA$ was performed in $\mathrm{G} 09$ at the M06-2X/6-31G(d) level of theory resulting in 101 structures. The Gaussian output file was used as input for autoDIAS. The structure parser method for scans and IRC calculations from G09 files implemented in autoDIAS does only extract converged structures. 81 converged structures were extracted, 20 nonconverged structures were ignored. Automated Distortion/Interaction Activation Strain analysis was performed and is shown in Figure 4. Fragments and the relevant $\mathrm{B}-\mathrm{N}$ bond was automatically detected by autoDIAS. Input and geometries were fetched in 23 seconds 309 ms, G09 input files for 81 structures (243 files) were prepared in 1 second $119 \mathrm{~ms}$. Total energy, total distortion energy and interaction energy is plotted against the B-N distance. Note the missing points especially around $3 \AA$ resulting from non-converged structures. This analysis shows that stabilizing interactions occur much earlier when moving the Lewis acid/base pair together, starting at a distance over $4 \AA$ while distortion energies do not contribute significantly down to a distance of $2.5 \AA$.

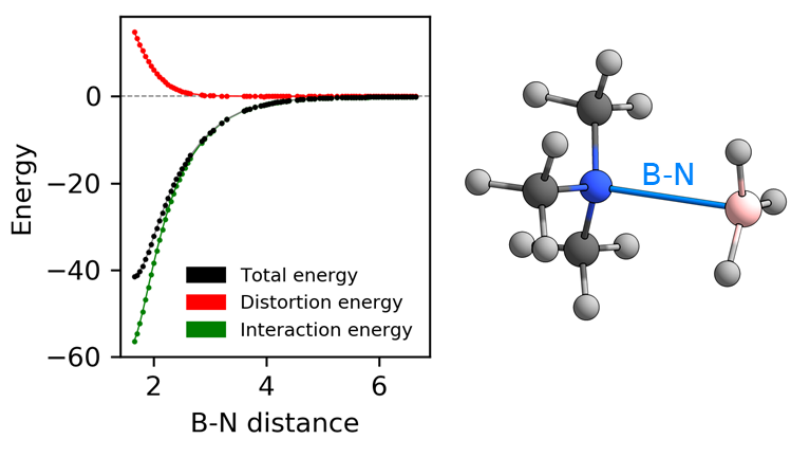

Figure 4. DIAS analysis for a scan along the BN bond distance of the trimethylamine-borane complex. Energy in $\mathrm{kcal} / \mathrm{mol}$, distance in $\AA$.

IRC 1

An IRC calculation of the archetypal Diels-Alder reaction between acrolein and 1,3-butadiene was performed in G09 at the M06-2X/6-31G(d) level of theory. The calculation resulted in 589 points along the IRC of which 90 were used for the DIAS analysis using the reduce option (RMSD threshold $=0.025$ ) implemented in autoDIAS. The input file and structures were imported within 2 seconds and $651 \mathrm{~ms}$, Gaussian input files for 90 structures were prepared in 762 ms. Fragments and both forming bonds were automatically and correctly detected. Total energy, distortion energies of both fragments, total distortion energy and interaction action are plotted against the shorter forming bond (Figure 5). 

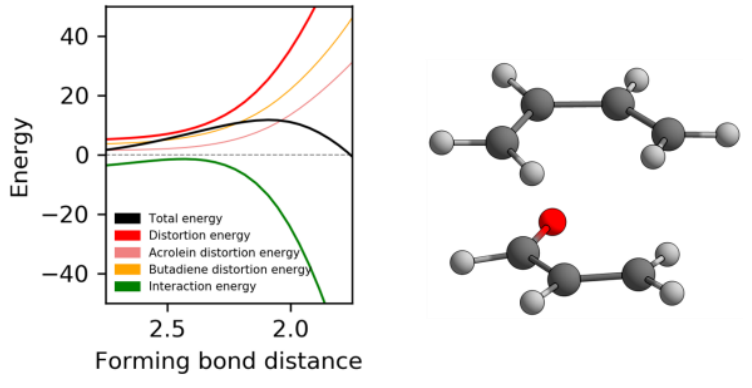

Figure 5. DIAS analysis of the Diels-Alder reaction between acrolein and 1,3-butadiene. Transition state geometry shown on the right. Energy in $\mathrm{kcal} / \mathrm{mol}$, distance in $\AA$.

\section{Technical Details}

autoDIAS was written in Python for use under Linux. It can therefore be used on most local machines and HPC clusters. A version for Windows 10 was not prepared since the availability of the Ubuntu subsystem under Windows (WSL) allows running Linux software natively on Windows computers. autoDIAS is compatible and was tested with Python versions 2.6 and higher, however, we recommend the use of Python 3.5 or higher. autoDIAS was tested on the Windows 10 Ubuntu subsystem on a personal computer, on the Hoffman2 cluster at UCLA and the Vienna Scientific Cluster 3 (VSC3, Vienna, Austria). The most up-to-date version is available for download on GitHub. Gaussian 09 Revision D.01, ${ }^{44}$ Gaussian 16 Revision A.03, ${ }^{45}$ ORCA 4.0.1.2, ${ }^{46}$ Q-Chem $4.3^{47}$ and NWChem $6.6^{48}$ were used as drivers. Chemcraft 1.8 was used for visualization. ${ }^{49}$

To use autoDIAS one must provide two input files; 1 ) A file containing molecular geometries, at the moment single or multi structure $\mathrm{XYZ}$ files or Gaussian output files and 2) an input file containing the needed information to perform the DIAS analysis. This includes the name of the file containing the structures, details about the structures and fragments, such as charge and multiplicity, information about the analysis such as what bonds, angles or dihedral angles should be extracted, information about further settings such as which files to keep after the analysis and information about how calculations using the selected driver are performed. The last section allows for easy adaption of other computational chemistry software packages and the use on different systems with different commands for running these calculations. An example of an input file with detailed descriptions of the settings can be found in the supporting information and is included in the software package.

autoDIAS accepts XYZ files and Gaussian output files as input. Possible Gaussian files include output from single point, frequency analysis, geometry optimizations, combined geometry optimization frequency analysis, IRC and scan jobs. In case of IRC and scan jobs only converged points are extracted. This can be circumvented by converting the output file in a XYZ file containing all structures and using this as input for autoDIAS, however, using nonconverged structures is not advised. Figure 6 shows the extracted (black) and omitted (red) points for scan 1.

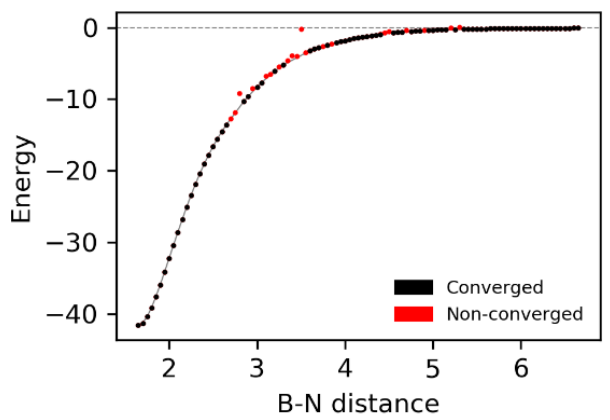

Figure 6. Visualization of the omitted, non-converged points (red) for the electronic energy of scan 1 . Energy in $\mathrm{kcal} / \mathrm{mol}$, distances in $\AA$.

IRC calculations in Gaussian which are performed in both directions start at the provided transition state geometry and are first propagated in one direction and then propagated in the other direction starting from the transition state again. However, this means that extracted geometries do not represent a continuous progress along the reaction coordinate. The reorder setting provides a tool that corrects this error but can potentially be used in any cases in which two continuous series of geometries are stitched together in an incorrect orientation. This is realized by calculating the RMSD, using the Procrustes analysis on centroid centered structures, between any two consecutive structures. If at least one of the RMSDs between two geometries is more 
than three times the average RMSD the point with the biggest RMSD is detected as the cut between the two separate parts. RMSD calculations are then performed between the first and last points of each part and orders within the parts are reversed to minimize the structural difference at the cut (Figure 7).
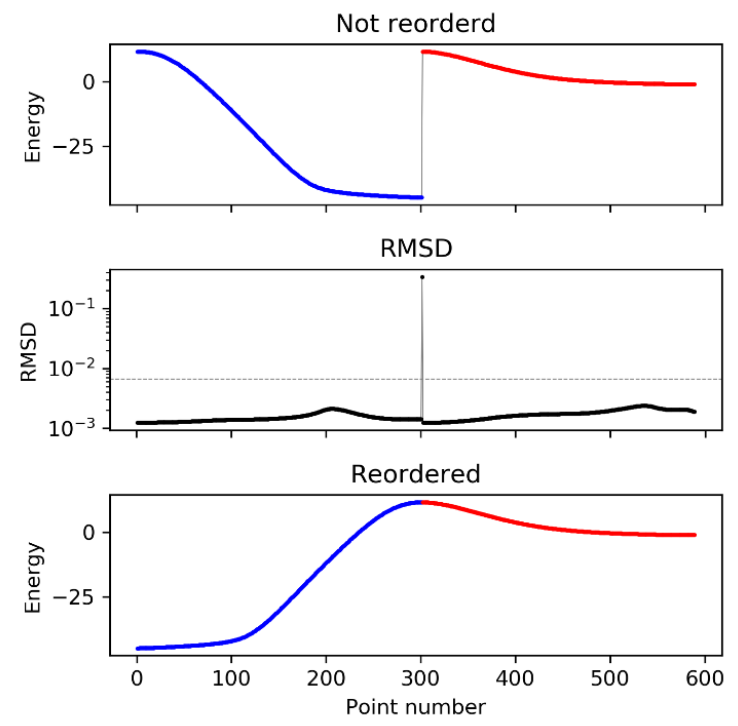

Figure 7. Reordering of the points in IRC 1. Energy in $\mathrm{kcal} / \mathrm{mol}$. The dashed line in the RMSD plot represents the threshold of 3 times the average RMSD.

From the provided input structures, fragments are produced by slicing the geometries according to provided lists of atom numbers in each fragment. Since providing such lists can be cumbersome, especially for larger molecules, autoDIAS provides two utilities to reduce the needed input. The first method requires the input of the atom list for one fragment, the atom list for the other fragment is then automatically determined. The second method is the completely automated determination of two fragments by providing no input in this regard. Fragment 1 will be the one including the first atom in the structure, which is helpful for naming fragments and providing relaxed geometry energies for calculation of distortion and relative energies. The automatic fragment detection will fail if only structures with lower fragment numbers than 2 are provided. If more than two fragments are detected some will be combined to result in two fragments. Fragments are determined by connecting atoms through bonds and grouping continuously connected atoms. Bonds are defined by using covalent radii ${ }^{50}$ for each atom type plus a $25 \%$ overhead. In case of an analysis of multiple structures where it might occur that two fragments become one over the progress of the analyzed coordinate the two fragments that occur most often are chosen.

Calculations containing a big amount of structures can be computationally expensive. The reduce option allows for a reduction of overall number of structures to reduce the calculation time. This utility is inspired by a similar tool used in the Bickelhaupt group in combination with PyFrag. Starting by selecting the first point, consecutive points are deleted if the RMSD between that structure and the currently selected one is below a provided threshold. Once a structure is reached that differs by more than threshold this structure is selected, and the progress is repeated until the last structure is reached. Figure 8 shows the effect of this reduction on the electronic energy of the analysis of an IRC calculation using G09 of Diels-Alder reaction IRC 1. This calculation was performed using the IRC=(stepsize=1) setting in G09 to produce a large amount of points along the IRC, resulting in 589 points. Using reduce in combination with a RMSD threshold of $0.005 \AA$ reduces the amount of points by $71.5 \%$. Using $0.01 \AA$ as threshold the amount is reduced by another $13.2 \%$ down to $15.3 \%$ of total points. A threshold of $0.025 \AA$ A reduces the amount to only 39 points $(6.6 \%)$ while still providing a good image of the energy progress along the reaction coordinate.
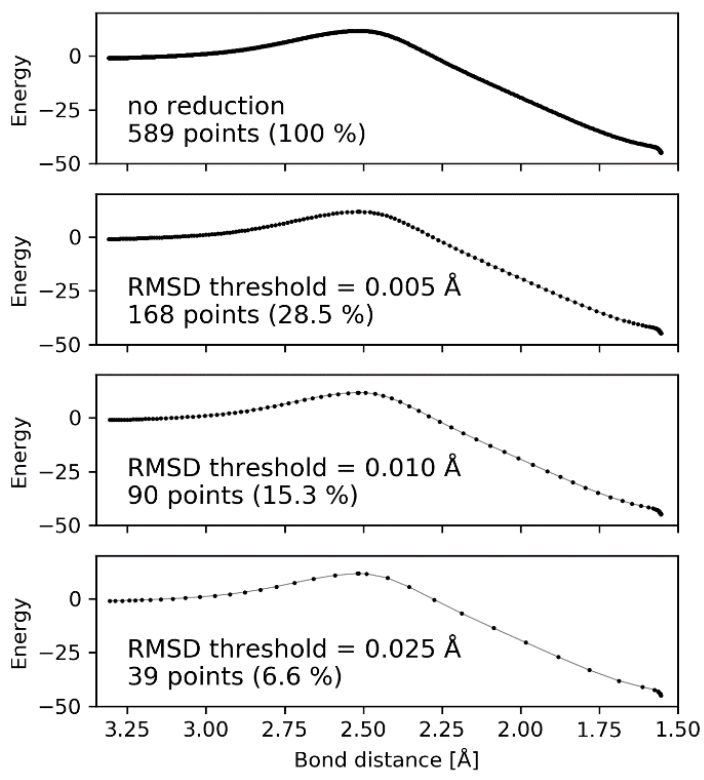
Figure 8. Comparison between the result using different levels of the reduce utility in autoDIAS for IRC 1. Electronic energy in $\mathrm{kcal} / \mathrm{mol}$ is shown.

Analysis of the performed calculation can be requested by using the analysis keyword (Analysis = YES). Not using this option is necessary in case of a driver or performed calculation for which no parser for the energy is implemented in autoDIAS. Extraction of geometric parameters is important for multi structure DIAs, allowing for analysis and plotting along selected coordinates. autoDIAS allows for extraction of bond lengths, angles and dihedral angles. Parameters which should be extracted can be specified in the input file. autoDIAS allows for automatic detection of bonds. This is requested by using the keyword "auto" in the geometry bond section of the input file, providing no input in this section or providing only incorrect input. This automated process does only work correctly in case of multiple geometry calculations and is performed by calculating the changes in bond orders, determined as described for the automated fragment detection. The two bonds that change the fewest times but at least once over the progress of the scan/reaction coordinate are selected. This crude method provided good results in all tested cases. Bonds were automatically and correctly detected in all presented cases. Bond angles are requested by providing 3 atoms in the angle input section and dihedral angles are requested by specifying 4 atoms. Invalid requests are ignored.

Several further settings are available. These include the option to delete keep all calculation input files (Keep input files $=$ YES), produce $X Y Z$ files for all structures, fragments, and a file including all structures in one file (Keep xyz = YES), and keep the log file providing information about the performed DIAS analysis (Keep log file $=$ YES).

In addition, the prepare only mode requests reading of the input file and preparation of the input and *.xyz files. If necessary automatic detection of fragments, reduction of structures, and reordering is also performed. This mode allows for determination if the automated fragment and bond detection works correctly or if the chosen RMSD threshold for the reduce option reduces the number of calculated points to a reasonable amount. It can also be used to prepare the needed input file before running the needed calculations on a different system and/or in parallel.

The analysis only mode requests analysis of the previously generated output files only. This can be useful to add further geometric parameters to the analysis or add previously missing fragment energies. It can be requested using the "-a" flag. This mode ignores all input regarding the execution of the prepared input files and prepares an DIA analysis file equal to that produced during normal autoDIAS operation. It is to note that this modus still parses the structure input file and performs any requested changes (i.e. reduction of points and reordering) to fetch geometry data.

The user needs to provide a template for the input for the single point calculation for the chosen driver in the layout section. Wildcards can be used for filename, charge, multiplicity and (cartesian) coordinates. In addition, the user has to provide the file extensions for input and output files for these calculations, and the syntax for performing the calculation on the given computer system in the run_job section of the input file, using Wildcards for input and output file names.

Minimal input for an analysis using autoDIAS are the file name of the file containing the structures, charges and multiplicities and information about running the calculations (layout and run_job sections). In addition, input of fragment energies is advised.

Output is provided in a text-based file named <jobname>_DIAS.txt listing all requested geometric parameters and calculated energies. The numpy "genfromtxt" and the MS Excel "text to columns" functions can be used to convert the data into an array that can be used for further manipulation or plotting. An additional log file is provided named <jobname>_log.txt containing information about the analysis and timing, this file is deleted after successful completion in case the keep log option is set to NO. Input and output files from energy calculations and xyz files are produced/kept if the corresponding options are set. 


\section{Performance}

Performance of autoDIAS was investigated using the Windows 10 Ubuntu subsystem and Python 3.7.1 on a Dell XPS13 9370 equipped with an Intel i7-8550u CPU and 16GB of memory. Several operations within autoDIAS could be optimized for speed. However, performance examples shown in Table 1 demonstrate that operations even for hundreds of structures only take some seconds. Reordering of structures adds approx. $10 \%$ time to the time spend in producing input structures. The time for parsing the structures which includes splitting them into fragments and reading the input file strongly depends on the nature and size of the input file while the time for producing the input files and performing analysis depends on the amount of structures. On average $120 \pm 40$ input files were produced per second and $760 \pm 170$ files were analyzed per second. Compared to the amount of time needed to perform energy calculations time spend in input file parsing, input file creation and analysis is neglectable.

Figure 8. Performance demonstration.

\begin{tabular}{|c|c|c|c|c|c|c|c|c|c|c|}
\hline & $\begin{array}{l}\text { File } \\
\text { size }\end{array}$ & File type & $\begin{array}{c}\text { Converged } \\
\text { structures }\end{array}$ & Reorder & Reduce & $\begin{array}{c}\text { Resulting } \\
\text { points }\end{array}$ & $\begin{array}{l}\text { Used } \\
\text { driver }\end{array}$ & $\begin{array}{c}\text { Time } \\
\text { for } \\
\text { parsing } \\
{[\mathrm{s}]}\end{array}$ & $\begin{array}{c}\text { Time for } \\
\text { producing } \\
\text { input files } \\
{[\mathrm{s}]}\end{array}$ & $\begin{array}{c}\text { Time for } \\
\text { analysis } \\
{[\mathrm{s}]}\end{array}$ \\
\hline \multirow{2}{*}{ Scan 1} & $66 \mathrm{MB}$ & G09 (scan) & 81 & No & No & 81 & \multirow{12}{*}{ G09 } & 5.071 & 1.756 & 0.282 \\
\hline & $84 \mathrm{~KB}$ & XYZ (multi) & 101 & No & No & 101 & & 0.137 & 2.216 & 0.324 \\
\hline \multirow{6}{*}{ IRC 1} & \multirow{6}{*}{$5.5 \mathrm{MB}$} & \multirow{6}{*}{ G09 (IRC) } & \multirow{6}{*}{589} & Yes & No & 589 & & 0.935 & 12.488 & 3.215 \\
\hline & & & & No & No & 589 & & 0.945 & 11.499 & 3.222 \\
\hline & & & & Yes & Yes $(0.005)$ & 168 & & 0.936 & 3.277 & 0.607 \\
\hline & & & & Yes & Yes $(0.01)$ & 90 & & 0.973 & 1.786 & 0.330 \\
\hline & & & & No & Yes $(0.01)$ & 90 & & 0.974 & 1.582 & 0.313 \\
\hline & & & & Yes & Yes $(0.025)$ & 39 & & 0.983 & 0.806 & 0.103 \\
\hline \multirow{7}{*}{$\begin{array}{c}\text { Reaction } \\
1\end{array}$} & $60 \mathrm{~KB}$ & G09 (SP) & 1 & No & No & 1 & & 0.020 & 0.054 & 0.004 \\
\hline & $605 \mathrm{~KB}$ & G09 (Opt) & 1 & No & No & 1 & & 0.060 & 0.035 & 0.005 \\
\hline & $332 \mathrm{~KB}$ & G09 (Freq) & 1 & No & No & 1 & & 0.018 & 0.036 & 0.004 \\
\hline & $977 \mathrm{~KB}$ & G09 (Opt Freq) & 1 & No & No & 1 & & 0.060 & 0.040 & 0.004 \\
\hline & \multirow{3}{*}{$3 \mathrm{~KB}$} & \multirow{3}{*}{ XYZ (single) } & \multirow{3}{*}{1} & \multirow{3}{*}{ No } & \multirow{3}{*}{ No } & \multirow{3}{*}{1} & ORCA & 0.010 & 0.038 & 0.005 \\
\hline & & & & & & & NWChem & 0.011 & 0.035 & 0.005 \\
\hline & & & & & & & Q-Chem & 0.013 & 0.038 & 0.003 \\
\hline
\end{tabular}

\section{Conclusions}

We present autoDIAS, a python tool for the automated Distortion/Interaction Activation Strain analysis. This tool allows for the automated setup, performance and analysis of a DIAS analysis, even for big amount of structures. autoDIAS is easy to use, high performing, requires little input, can be used with many electronic structure programs and even allows for a Distortion/Interaction
Activation Strain analysis along a two, or more, dimensional potential energy surface. We are confident that autoDIAS will be a useful tool for computational chemists who wish to analyze energy barriers and surfaces using the Distortion/Interaction Activation Strain analysis.

\section{Acknowledgments}

We are grateful to the Austrian Science Fund (FWF, J 4216-N28) and the 
Hochschuljubiläumsstiftung of the city of Vienna (H-331849/2018) for financial support of this research. Computer time at Hoffman2 was provided by the UCLA Institute for Digital Research and Education (IDRE). Parts of this work were performed on the Vienna Scientific Cluster.

Keywords: Computational chemistry, Bond theory, Distortion, Strain, Software

((Additional Supporting Information may be found in the online version of this article.))

\section{References and Notes}

1. Ess, D. H.; Houk, K. N. Journal of the American Chemical Society 2007, 129(35), 10646-10647.

2. Fernández, I.; Bickelhaupt, F. M. Chem Soc Rev 2014, 43(14), 4953-4967.

3. Bickelhaupt, F. M. Journal of Computational Chemistry 1999, 20(1), 114-128.

4. Bickelhaupt, F. M.; Houk, K. N.

Angewandte Chemie International Edition 2017, 56(34), 10070-10086.

5. Morokuma, K. The Journal of Chemical Physics 1971, 55(3), 1236-1244.

6. Yu, S.; de Bruijn, H. M.; Svatunek, D.; Hamlin, T. A.; Bickelhaupt, F. M. ChemistryOpen 2018, 7(12), 995-1004.

7. Svatunek, D.; Houszka, N.; Hamlin, T. A.; Bickelhaupt, F. M.; Mikula, H. Chemistry - A European Journal 2019, 25(3), 754-758.

8. Riesco-Domínguez, A.; van de Wiel, J.; Hamlin, T. A.; van Beek, B.; Lindell, S. D.; BlancoAnia, D.; Bickelhaupt, F. M.; Rutjes, F. P. J. T. The Journal of Organic Chemistry 2018, 83(4), 1779-1789.

9. Mikula, H.; Kronister, S.; Svatunek, D.; Denk, C. Synlett 2018, 29(10), 1297-1302.

10. Liu, F.; Liang, Y.; Houk, K. N. Accounts of Chemical Research 2017, 50(9), 2297-2308.

11. Gold, B.; Aronoff, M. R.; Raines, R. T. Organic Letters 2016, 18(18), 4466-4469.

12. Escorihuela, J.; Das, A.; Looijen, W. J. E.; van Delft, F. L.; Aquino, A. J. A.; Lischka, H.;
Zuilhof, $\mathrm{H}$. The Journal of Organic Chemistry 2017, 83(1), 244-252.

13. Cabrera-Trujillo, J. J.; Fernández, I. Organic \& Biomolecular Chemistry 2019, 17(11), 2985-2991.

14. Fernández, I. European Journal of Organic Chemistry 2018, 2018(12), 1394-1402.

15. García-Rodeja, Y.; Fernández, I. The Journal of Organic Chemistry 2019, 84(7), 43304337.

16. Wang, Z.; Danovich, D.; Ramanan, R.; Shaik, S. Journal of the American Chemical Society 2018, 140(41), 13350-13359.

17. Gomes, G. d. P.; Loginova, Y.; Vatsadze, S. Z.; Alabugin, I. V. Journal of the American Chemical Society 2018, 140(43), 14272-14288.

18. Schoenebeck, F.; Ess, D. H.; Jones, G. O.; Houk, K. N. Journal of the American Chemical Society 2009, 131(23), 8121-8133.

19. Fernández, I.; Bickelhaupt, F. M. Journal of Computational Chemistry 2012, 33(5), 509516.

20. Faza, O. N.; López, C. S.; Fernández, I. The Journal of Organic Chemistry 2013, 78(11), 5669-5676.

21. Vermeeren, P.; Sun, X.; Bickelhaupt, F. M. Scientific Reports 2018, 8(1).

22. Green, A. G.; Liu, P.; Merlic, C. A.; Houk, K. N. Journal of the American Chemical Society 2014, 136(12), 4575-4583.

23. Schwarz, H.; Shaik, S.; Li, J. Journal of the American Chemical Society 2017, 139(48), 17201-17212.

24. Lu, G.; Liu, R. Y.; Yang, Y.; Fang, C.; Lambrecht, D. S.; Buchwald, S. L.; Liu, P. Journal of the American Chemical Society 2017, 139(46), 16548-16555.

25. Thomas, A. A.; Speck, K.; Kevlishvili, I.; Lu, Z.; Liu, P.; Buchwald, S. L. Journal of the American Chemical Society 2018, 140(42), 13976-13984.

26. Schoenebeck, F.; Houk, K. N. Journal of the American Chemical Society 2010, 132(8), 2496-2497.

27. Garcia, Y.; Schoenebeck, F.; Legault, C. Y.; Merlic, C. A.; Houk, K. N. Journal of the American Chemical Society 2009, 131(18), 6632-6639. 
28. Maji, R.; Mallojjala, S. C.; Wheeler, S. E. Chemical Society Reviews 2018, 47(4), 11421158.

29. Lu, T.; Porterfield, M. A.; Wheeler, S. E. Organic Letters 2012, 14(20), 5310-5313.

30. Maji, R.; Champagne, P. A.; Houk, K. N.; Wheeler, S. E. ACS Catalysis 2017, 7(10), 73327339.

31. Seguin, T. J.; Wheeler, S. E. ACS Catalysis 2016, 6(10), 7222-7228.

32. Rutledge, K. M.; Hamlin, T. A.; Baldisseri, D. M.; Bickelhaupt, F. M.; Peczuh, M. W. Chemistry - An Asian Journal 2017, 12(19), 2623-2633.

33. Kubelka, J.; Bickelhaupt, F. M. The Journal of Physical Chemistry A 2017, 121(4), 885-891.

34. Hamlin, T. A.; van Beek, B.; Wolters, L. P.; Bickelhaupt, F. M. Chemistry - A European Journal 2018, 24(22), 5927-5938.

35. Mitchell, D. J.; Schlegel, H. B.; Shaik, S. S.; Wolfe, S. Canadian Journal of Chemistry 1985, 63(7), 1642-1648.

36. Hamlin, T. A.; Svatunek, D.; Yu, S.; Ridder, L.; Infante, I.; Visscher, L.; Bickelhaupt, F. M. European Journal of Organic Chemistry 2019, 2019(2-3), 378-386.

37. Levandowski, B. J.; Svatunek, D.; Sohr, B.; Mikula, H.; Houk, K. N. Journal of the American Chemical Society 2019, 141(6), 22242227.

38. Zeist, W.-J. V.; Guerra, C. F.; Bickelhaupt, F. M. Journal of Computational Chemistry 2008, 29(2), 312-315.

39. te Velde, G.; Bickelhaupt, F. M.; Baerends, E. J.; Fonseca Guerra, C.; van Gisbergen, S. J. A.; Snijders, J. G.; Ziegler, T. Journal of Computational Chemistry 2001, 22(9), 931-967.

40. Baerends, E. J.; Ziegler, T.; Atkins, A. J.; Autschbach, J.; Bashford, D.; Baseggio, O.; Brces, A.; Bickelhaupt, F. M.; Bo, C.; Boerritger, P. M.; Cavallo, L.; Daul, C.; Chong, D. P.; Chulhai, D. V.; Deng, L.; Dickson, R. M.; Dieterich, J. M.; Ellis, D. E.; van Faassen, M.; Ghysels, A.; Giammona, A.; van Gisbergen, S. J. A.; Goez, A.; Gtz, A. W.; Gusarov, S.; Harris, F. E.; van den Hoek, P.; Hu, Z.; Jacob, C. R.; Jacobsen, H.;
Jensen, L.; Joubert, L.; Kaminski, J. W.; van Kessel, G.; Knig, C.; Kootstra, F.; Kovalenko, A.; Krykunov, M.; van Lenthe, E.; McCormack, D. A.; Michalak, A.; Mitoraj, M.; Morton, S. M.; Neugebauer, J.; Nicu, V. P.; Noodleman, L.; Osinga, V. P.; Patchkovskii, S.; Pavanello, M.; Peeples, C. A.; Philipsen, P. H. T.; Post, D.; Pye, C. C.; Ramanantoanina, H.; Ramos, P.; Ravenek, W.; Rodrguez, J. I.; Ros, P.; Rger, R.; Schipper, P. R. T.; Schlns, D.; van Schoot, H.; Schreckenbach, G.; Seldenthuis, J. S.; Seth, M.; Snijders, J. G.; Sol.

41. Laloo, J. Z. A.; Laloo, N.; Rhyman, L.; Ramasami, P. Journal of Computer-Aided Molecular Design 2017, 31(7), 667-673.

42. Laloo, J. Z. A.; Savoo, N.; Laloo, N.; Rhyman, L.; Ramasami, P. Journal of Computational Chemistry 2019, 40(3), 619-624. 43.

44. Frisch, M. J.; Trucks, G. W.; Schlegel, H. B.; Scuseria, G. E.; Robb, M. A.; Cheeseman, J. R.; Scalmani, G.; Barone, V.; Mennucci, B.; Petersson, G. A.; Nakatsuji, H.; Caricato, M.; Li, X.; Hratchian, H. P.; Izmaylov, A. F.; Bloino, J.; Zheng, G.; Sonnenberg, J. L.; Hada, M.; Ehara, M.; Toyota, K.; Fukuda, R.; Hasegawa, J.; Ishida, M.; Nakajima, T.; Honda, Y.; Kitao, O.; Nakai, H.; Vreven, T.; Montgomery, J. A.; Peralta, J. E.; Ogliaro, F.; Bearpark, M.; Heyd, J. J.; Brothers, E.; Kudin, K. N.; Staroverov, V. N.; Kobayashi, R.; Normand, J.; Raghavachari, K.; Rendell, A.; Burant, J. C.; lyengar, S. S.; Tomasi, J.; Cossi, M.; Rega, N.; Millam, J. M.; Klene, M.; Knox, J. E.; Cross, J. B.; Bakken, V.; Adamo, C.; Jaramillo, J.; Gomperts, R.; Stratmann, R. E.; Yazyev, O.; Austin, A. J.; Cammi, R.; Pomelli, C.; Ochterski, J. W.; Martin, R. L.; Morokuma, K.; Zakrzewski, V. G.; Voth, G. A.; Salvador, P.; Dannenberg, J. J.; Dapprich, S.; Daniels, A. D.; Farkas; Foresman, J. B.; Ortiz, J. V.; Cioslowski, J.; Fox, D. J.: Wallingford CT, 2009.

45. Frisch, M. J.; Trucks, G. W.; Schlegel, H. B.; Scuseria, G. E.; Robb, M. A.; Cheeseman, J. R.; Scalmani, G.; Barone, V.; Petersson, G. A.; Nakatsuji, H.; Li, X.; Caricato, M.; Marenich, A. V.; Bloino, J.; Janesko, B. G.; Gomperts, R.; Mennucci, B.; Hratchian, H. P.; Ortiz, J. V.; Izmaylov, A. F.; Sonnenberg, J. L.; Williams; 
Ding, F.; Lipparini, F.; Egidi, F.; Goings, J.; Peng, B.; Petrone, A.; Henderson, T.; Ranasinghe, D.; Zakrzewski, V. G.; Gao, J.; Rega, N.; Zheng, G.; Liang, W.; Hada, M.; Ehara, M.; Toyota, K.; Fukuda, R.; Hasegawa, J.; Ishida, M.; Nakajima, T.; Honda, Y.; Kitao, O.; Nakai, H.; Vreven, T.; Throssell, K.; Montgomery Jr., J. A.; Peralta, J. E.; Ogliaro, F.; Bearpark, M. J.; Heyd, J. J.; Brothers, E. N.; Kudin, K. N.; Staroverov, V. N.; Keith, T. A.; Kobayashi, R.; Normand, J.; Raghavachari, K.; Rendell, A. P.; Burant, J. C.; Iyengar, S. S.; Tomasi, J.; Cossi, M.; Millam, J. M.; Klene, M.; Adamo, C.; Cammi, R.; Ochterski, J. W.; Martin, R. L.; Morokuma, K.; Farkas, O.; Foresman, J. B.; Fox, D. J.: Wallingford, CT, 2016.

46. Neese, F. Wiley Interdisciplinary Reviews: Computational Molecular Science 2018, 8(1).

47. Shao, Y.; Gan, Z.; Epifanovsky, E.; Gilbert, A. T. B.; Wormit, M.; Kussmann, J.; Lange, A. W.; Behn, A.; Deng, J.; Feng, X.; Ghosh, D.; Goldey, M.; Horn, P. R.; Jacobson, L. D.; Kaliman, I.; Khaliullin, R. Z.; Kuś, T.; Landau, A.; Liu, J.; Proynov, E. I.; Rhee, Y. M.; Richard, R. M.; Rohrdanz, M. A.; Steele, R. P.; Sundstrom, E. J.; Woodcock, H. L.; Zimmerman, P. M.; Zuev, D.; Albrecht, B.; Alguire, E.; Austin, B.; Beran, G. J. O.; Bernard, Y. A.; Berquist, E.; Brandhorst, K.; Bravaya, K. B.; Brown, S. T.; Casanova, D.; Chang, C.-M.; Chen, Y.; Chien, S. H.; Closser, K. D.; Crittenden, D. L.; Diedenhofen, M.; DiStasio, R. A.; Do, H.; Dutoi, A. D.; Edgar, R. G.; Fatehi, S.; Fusti-Molnar, L.; Ghysels, A.; GolubevaZadorozhnaya, A.; Gomes, J.; Hanson-Heine, M. W. D.; Harbach, P. H. P.; Hauser, A. W.; Hohenstein, E. G.; Holden, Z. C.; Jagau, T.-C.; Ji, H.; Kaduk, B.; Khistyaev, K.; Kim, J.; Kim, J.; King, R. A.; Klunzinger, P.; Kosenkov, D.; Kowalczyk,
T.; Krauter, C. M.; Lao, K. U.; Laurent, A. D.; Lawler, K. V.; Levchenko, S. V.; Lin, C. Y.; Liu, F.; Livshits, E.; Lochan, R. C.; Luenser, A.; Manohar, P.; Manzer, S. F.; Mao, S.-P.; Mardirossian, N.; Marenich, A. V.; Maurer, S. A.; Mayhall, N. J.; Neuscamman, E.; Oana, C. M.; Olivares-Amaya, R.; O’Neill, D. P.; Parkhill, J. A.; Perrine, T. M.; Peverati, R.; Prociuk, A.; Rehn, D. R.; Rosta, E.; Russ, N. J.; Sharada, S. M.; Sharma, S.; Small, D. W.; Sodt, A.; Stein, T.; Stück, D.; Su, Y.-C.; Thom, A. J. W.; Tsuchimochi, T.; Vanovschi, V.; Vogt, L.; Vydrov, O.; Wang, T.; Watson, M. A.; Wenzel, J.; White, A.; Williams, C. F.; Yang, J.; Yeganeh, S.; Yost, S. R.; You, Z.-Q.; Zhang, I. Y.; Zhang, X.; Zhao, Y.; Brooks, B. R.; Chan, G. K. L.; Chipman, D. M.; Cramer, C. J.; Goddard, W. A.; Gordon, M. S.; Hehre, W. J.; Klamt, A.; Schaefer, H. F.; Schmidt, M. W.; Sherrill, C. D.; Truhlar, D. G.; Warshel, A.; Xu, X.; Aspuru-Guzik, A.; Baer, R.; Bell, A. T.; Besley, N. A.; Chai, J.-D.; Dreuw, A.; Dunietz, B. D.; Furlani, T. R.; Gwaltney, S. R.; Hsu, C.-P.; Jung, Y.; Kong, J.; Lambrecht, D. S.; Liang, W.; Ochsenfeld, C.; Rassolov, V. A.; Slipchenko, L. V.; Subotnik, J. E.; Van Voorhis, T.; Herbert, J. M.; Krylov, A. I.; Gill, P. M. W.; HeadGordon, M. Molecular Physics 2014, 113(2), 184-215.

48. Valiev, M.; Bylaska, E. J.; Govind, N.; Kowalski, K.; Straatsma, T. P.; Van Dam, H. J. J.; Wang, D.; Nieplocha, J.; Apra, E.; Windus, T. L.; de Jong, W. A. Computer Physics Communications 2010, 181(9), 1477-1489. 49. https://www.chemcraftprog.com. 50. Cordero, B.; Gómez, V.; Platero-Prats, A. E.; Revés, M.; Echeverría, J.; Cremades, E.; Barragán, F.; Alvarez, S. Dalton Transactions 2008(21). 


\section{GRAPHICAL ABSTRACT}

Dennis Svatunek and Kendall N. Houk

autoDIAS: a python tool for an automated Distortion/Interaction Activation Strain Analysis

Performing a Distortion/Interaction Activation Strain analysis can be cumbersome due to the potentially large amount of needed calculations which have to be prepared, run, and analyzed. autoDIAS is a python tool that helps automating this process while including a range of useful additional tools.

\section{autoDIAS}

Automated Distortion/Interaction Activation Strain Analysis

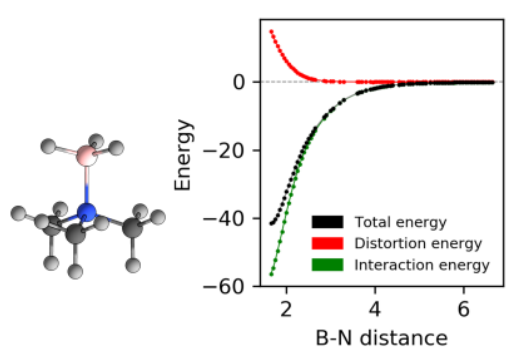




\section{autoDIAS}

\section{Automated Distortion/Interaction Activation Strain Analysis}
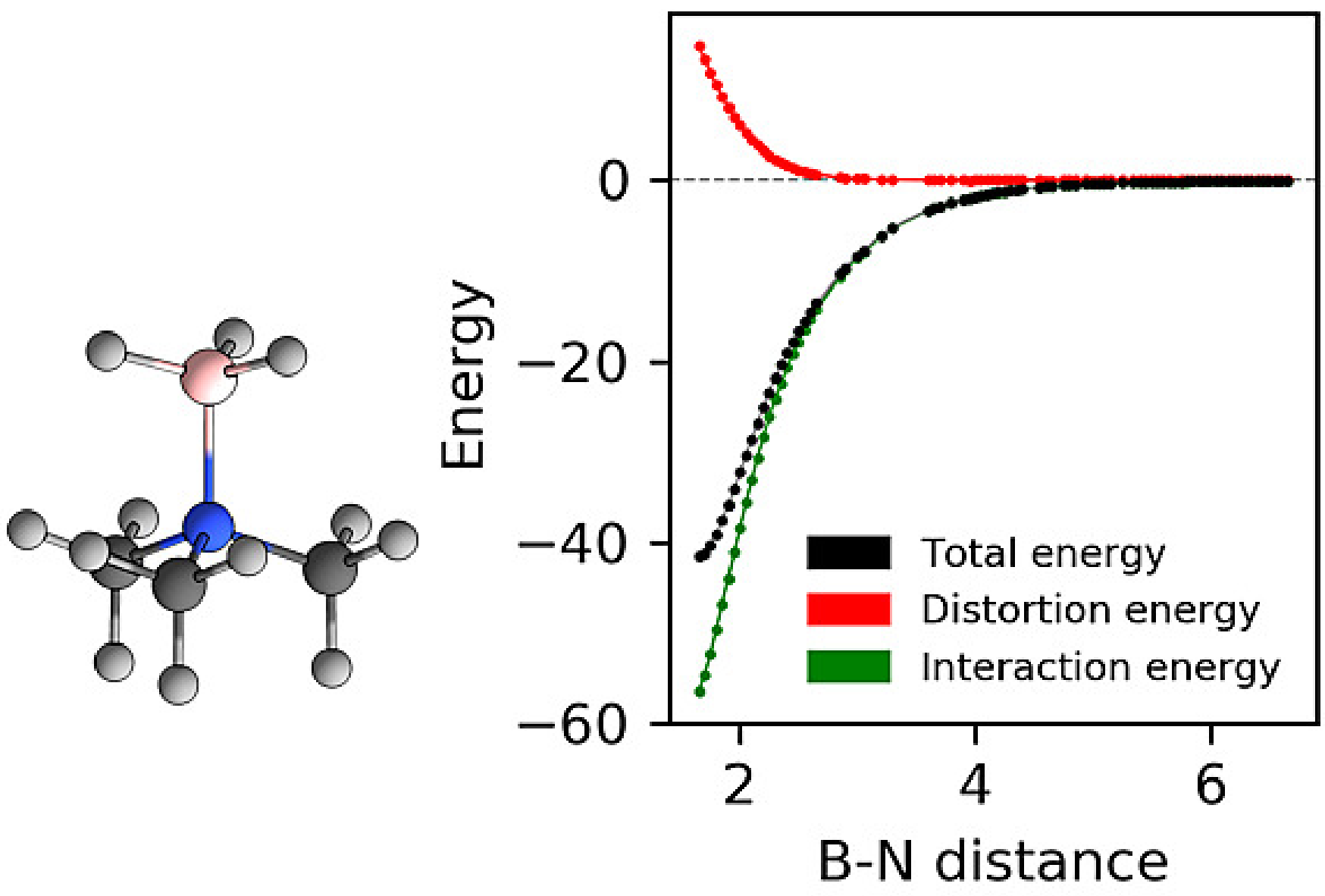


\section{Supporting Information}

\section{autoDIAS: a python tool for an automated Distortion/Interaction Activation Strain Analysis}

Dennis Svatunek* and Kendall N. Houk

Department of Chemistry and Biochemistry, University of California, Los Angeles, 90095, California, United States d.svatunek@chem.ucla.edu

Table of Contents

autoDIAS

autoDIAS.py

DIAS_inputparser

DIAS_reduce_reorder

DIAS_analysis

Settings input file

Log file 36

DIAS file 37

Plotting

Plotting of total, distortion and interaction energies for a single geometric parameter (used for Figure 5) 


\section{autoDIAS}

An up to date version of autoDIAS can be found on GitHub:

https://github.com/dsvatunek/autoDIAS

The autoDIA Stool consists of 4 python files:

autoDIAS.py

controls the analysis, produces input files for drivers, produces the log file

DIAS_inputparser parses settings and structures, checks input for wrong information, does the automated fragment and bond detection and prepares the fragment structures

DIAS_reduce_reorder responsible for the reduce and reorder functions of autoDIAS DIAS_analysis parses energies and geometric parameters, plots the result to the DIAS file 


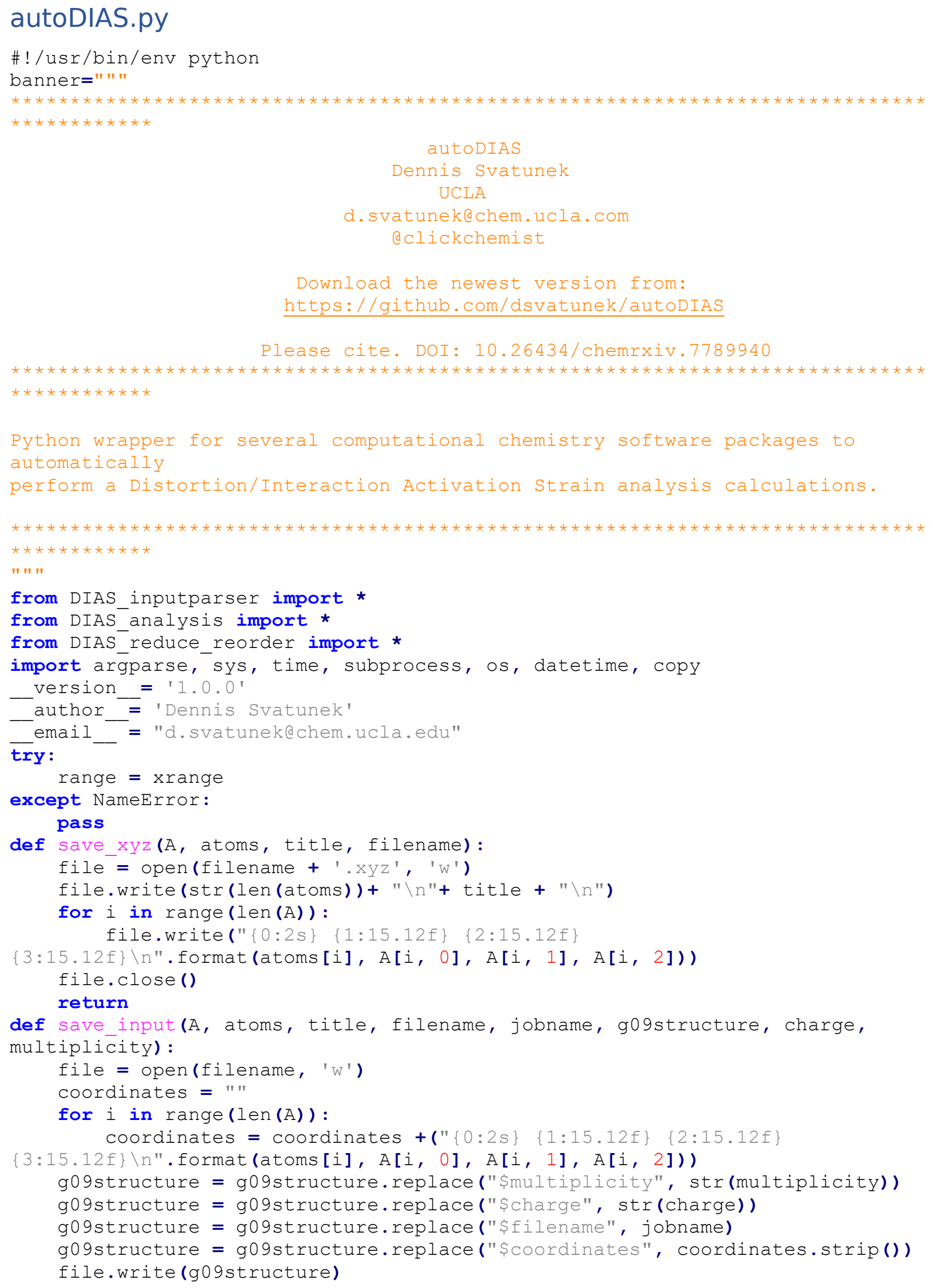


file.close ()

return

def molecular formula (atoms):

alphabetic_periodic_table = sorted(copy.deepcopy(periodic_table))

formula $=" "$

count $=[]$

for element in alphabetic_periodic_table:

if count [alphabetic_periodic table.index("C")] > 1 : formula $=$ formula $+{ }^{\prime} \mathrm{C}$ '

str(count [alphabetic_periodic_table.index("C")]) count[alphabetic_periodic_table.index("C")] = 0

elif count[alphabetic_periodic_table.index("C")] > 0 : formula = formula + ' $\mathrm{C}$ '

count[alphabetic_periodic_table.index("C")] = 0

if count[alphabetic_periodic_table.index("H")] > 1 : formula $=$ formula + 'H' $\overline{+}$

str(count [alphabetic_periodic_table.index("H")]) count[alphabetic_periōdic_table.index("H")] = 0

elif count[alphabetic_periodic_table.index("H")] > 0 : formula $=$ formula + 'H' count[alphabetic_periodic_table.index("H")] = 0

for $x$ in range (0, len(alphabetic_periodic_table)): if $\operatorname{count}[\mathrm{x}]==1$ : formula = formula + alphabetic_periodic_table[x]

elif count $[\mathrm{x}]>1$ :

formula = formula + alphabetic_periodic_table[x] + str(count $[\mathrm{x}])$ return formula

def $\operatorname{main}()$ :

parser = argparse.ArgumentParser(usage='(prog)s inp_file')

parser.add_argument('inp_file', metavar='Input file', type=str, help='Name of the input file')

parser.add_argument("-a", "--analysis", action='store_true', help='Analysis only', default=False)

if len (sys.argv) == 1 :

parser.print_help ()

sys.exit (1)

args = parser.parse_args()

overallstarttime=time.time()

starttime=time.time ()

settings, structures = parse_in(args.inp_file,args.analysis)

if args.analysis:

endtime=time.time ()

totaltime=str (endtime-starttime)

seconds=totaltime.split('.') [0]

milliseconds=float('0.'ttotaltime.split('.')[1])*1000

print('Processed input and parsed structures in $\{0\}$ seconds and

\{1:.0f\} ms'.format (seconds, float(milliseconds)))

if settings.reorder:

structures $=$ reorder (structures)

if settings.reduce:

structures $=$ reduce (structures, settings)

analysis_only (structures, settings)

return 


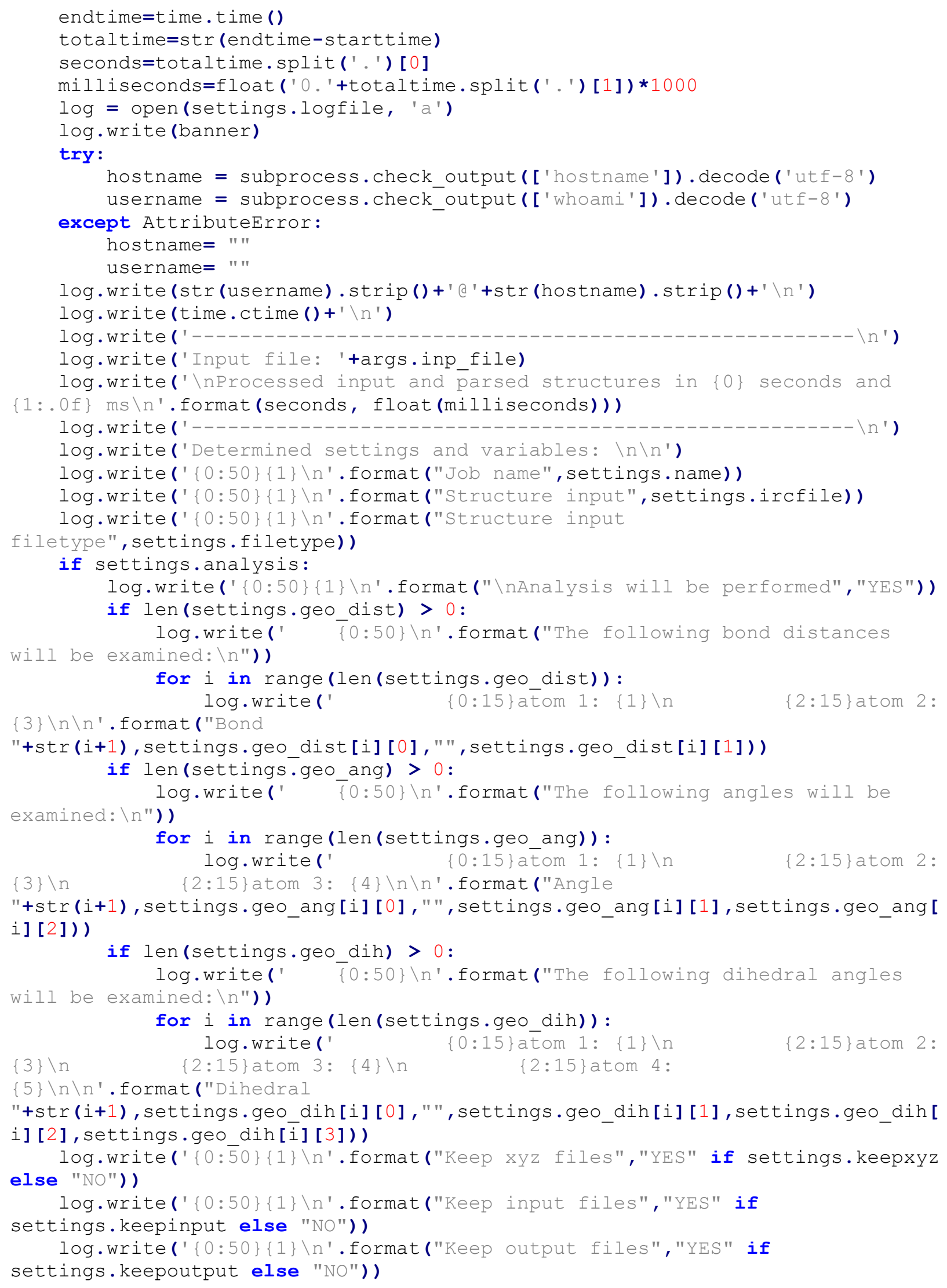


log.write('\{0:50\}\{1\}\n'.format("Keep this log file","YES" if settings.keeplog else "NO"))

log.write(' $\{0: 50\}\{1\} \backslash n$ '. format ("Reorder structures", "YES" if

settings.reorder else "NO"))

log.write('\{0:50\}\{1\}\n'.format("Reduce structures", "YES" if

settings.reduce else "NO"))

if settings.reduce:

log.write(' $\{0: 50\}\{1\} \backslash n$ '. format ("Threshold for reducing

structures", settings.reduce_tresh))

log.write(" $\{0: 50\}\{1\} \backslash n$ '. format ("Prepare input and exit", "YES" if

settings.prepareonly else "NO"))

formula = molecular formula (structures.atoms)

fragment 1 formula = molecular formula (structures.fraglatoms)

fragment2 formula = molecular_formula (structures.frag2atoms)

log.write (' \nstructure informātion \n\n')

log.write('Input structure has $\{0\}$ atoms with a molecular formula

of: $\{1:>15\} \backslash n \backslash n^{\prime}$. format (len(structures.atoms), formula))

log.write(' $\{0\}$ has $\{1\}$ atoms with a molecular formula

of: $\{2:>15\} \backslash n \backslash n^{\prime}$. format (settings.fraglname, len(structures.fraglatoms),

fragment 1 formula))

log.write(' $\{0\}$ has $\{1\}$ atoms with a molecular formula

of $:\{2:>15\} \backslash n \backslash n^{\prime}$. format (settings.frag2name, len(structures.frag2atoms),

fragment2 formula))

log.write('Complex has a charge and multiplicity of: $\{0\}$

$\{1\} \backslash n^{\prime}$.format (settings.charge, settings.multi))

log.write(' $\{0\}$ has a charge and multiplicity of: $\{1\}$

$\{2\} \backslash n^{\prime}$.format (settings.fraglname, settings.fraglcharge, settings.fraglmulti))

log.write('\{0\} has a charge and multiplicity of: $\{1\}$

$\{2\} \backslash n^{\prime}$.format (settings.frag2name, settings.frag2charge, settings.frag2multi))

log.write(' $\{0\}$ has an energy of: $\{1\} \backslash n$ '.format(settings.fraglname,

settings.fraglenergy))

log.write(' $\{0\}$ has an energy of: $\{1\} \backslash n$ '.format(settings.frag2name,

settings.frag2energy))

$\log \cdot$ write ('\n')

log.write(

$\log \cdot \mathrm{close}()$

if settings.reorder:

structures $=$ reorder (structures)

if settings.reduce:

structures $=$ reduce (structures, settings)

starttime=time.time ()

if settings.keepxyz:

if not os.path.exists (settings.name+' xyz'):

os.makedirs (settings.name+' $x y z^{\prime}$ )

for $x$ in range $(0$, len (structures.xyz)):

save_xyz(structures.xyz[x], structures.atoms,

structures.title $[\mathrm{x}]$, settings.name+' ${ }^{\prime} y z / \operatorname{complex}\{0: 04 \mathrm{~d}\}$ '. format (x+1)) save_xyz(structures.xyz_1[x],

structures.fraglätoms, settings.fraglname+' $\{0: 04 d\}$ '. format (x+1),

settings.namet' xyz/' +settings.fraglname+' $\{0: 04 d\}$ '. format (x+1)) save_xyz(structures.xyz_2[x],

structures.frag2atoms, settings.frag $\overline{2}$ name+' $\{0: 04 d\}$ '. format (x+1),

settings.namet' xyz/' +settings.frag2name+' $\{0: 04 d\}$ '. format (x+1))

os.system("cat $\{0\}$ xyz/complex*.xyz >

$\{0\}$ _xyz/complete.xyz".format (settings.name))

if not os.path.exists (settings.namet' input') :

os.makedirs (settings.namet'_input') 
if not os.path.exists (settings.name+'_output'):

os.makedirs (settings.namet'_output')

for $x$ in range $(0$, len(structures.xyz)):

save_input(structures.xyz[x], structures.atoms, structures.title[x],

settings.namét'_input/complex_\{0:04d\}.' .format $(x+1)+$ settings.input_file_exten sion, settings. name+'_output/Complex_\{0:04d\}'.format $(x+1)$,

settings.inputlayout, settings.chargè, settings.multi)

save_input(structures.xyz_1[x], structures.fraglatoms,

settings.fraḡinamet' $\{0: 04 d\}$ '. format $(x+1)$,

settings.namet' input/' +settings.fraglnamet' $\{0: 04 d\}$. '.format ( $\mathbf{x}+1)+$ settings.i

nput_file_extension,

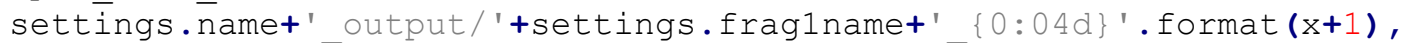

settings.inputlāyout, settings.fraglcharge, sēttings.fraglmulti) save input (structures.xyz_2[x], structures.frag2atoms,

settings.frā̄2name+' $\{0: 04 d\}\}^{\prime}$.format $(x+1)$,

settings.namet' input/' +settings.frag2name+' $\{0: 04 d\}$. '. format ( $\mathbf{x}+1)+$ settings.i

nput_file_extension,

settings.'namet' output/'+settings.frag2namet' $\{0: 04 d\}$ '.format (x+1),

settings.inputlayout, settings.frag2charge, settings.frag2multi)

endtime=time.time ()

totaltime=str (endtime-starttime)

seconds=totaltime.split(' ' ') [0]

milliseconds=float ('0.'ttotaltime.split('.')[1])*1000

$\log =$ open (settings.logfile, 'a')

log.write('Produced input files for $\{0\}$ structures in $\{1\}$ seconds and

$\{2: .0 f\} \mathrm{ms} \backslash \mathrm{n}^{\prime}$.format(len(structures.xyz), seconds, float(milliseconds)))

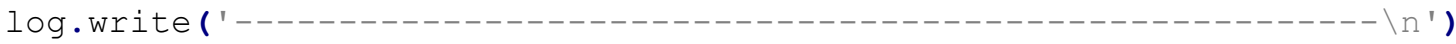

log.close ()

if settings.analysis and not settings.prepareonly:

create analysis file (settings)

$\log =\operatorname{open}($ settings.logfile, 'a')

if settings.prepareonly:

else:

log.write('No calculations requested! \n')

for $x$ in range $(0$, len (structures.xyz)):

command = settings.submit_setting.replace("\$input",

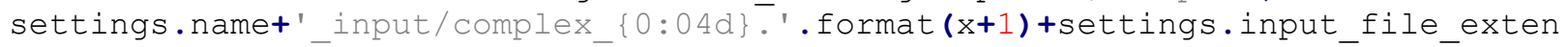
sion)

command = command.replace ("\$output",

settings.name+'_output/complex_\{0:04d\}.'.format $(x+1)+$ settings.output_file_ext ension)

log.write(time.ctime()+' $\{0:<30\}$ '. format (' $\backslash$ tstarting

$\{0:>15\}^{\prime}$.format ('complex $\{0: 04 d\}$ '. format $\left.\left.(x+1)\right)\right)$ )

$\log \cdot f \operatorname{lush}()$

starttime=time.time ()

os.system (command)

endtime=time.time ()

totaltime=endtime-starttime

log.write('\t finished after

$\{0: 0>15\} \backslash n^{\prime}$. format (str (datetime.timedelta (seconds=totaltime)))

$\log \cdot \mathrm{flush}()$ 


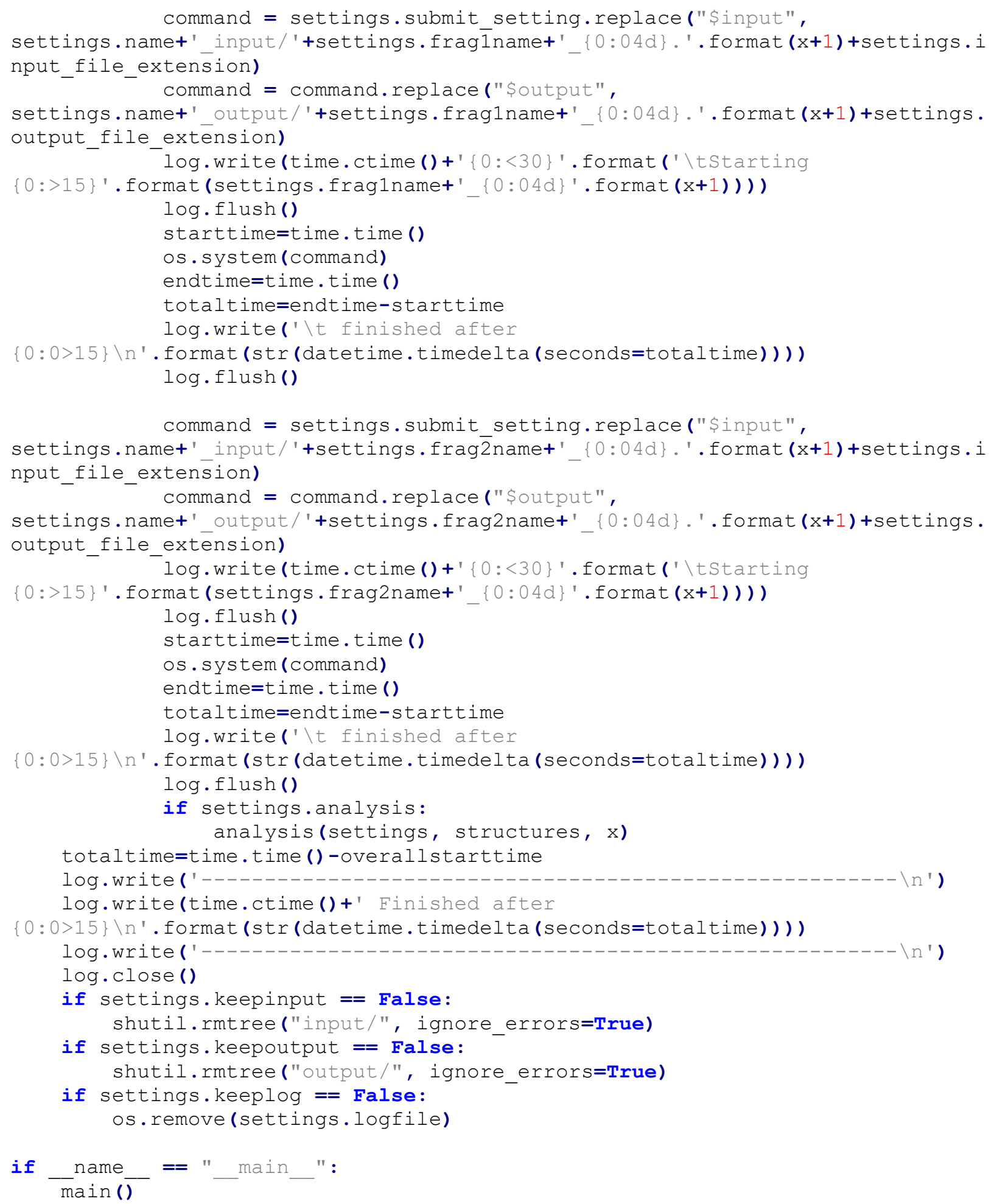




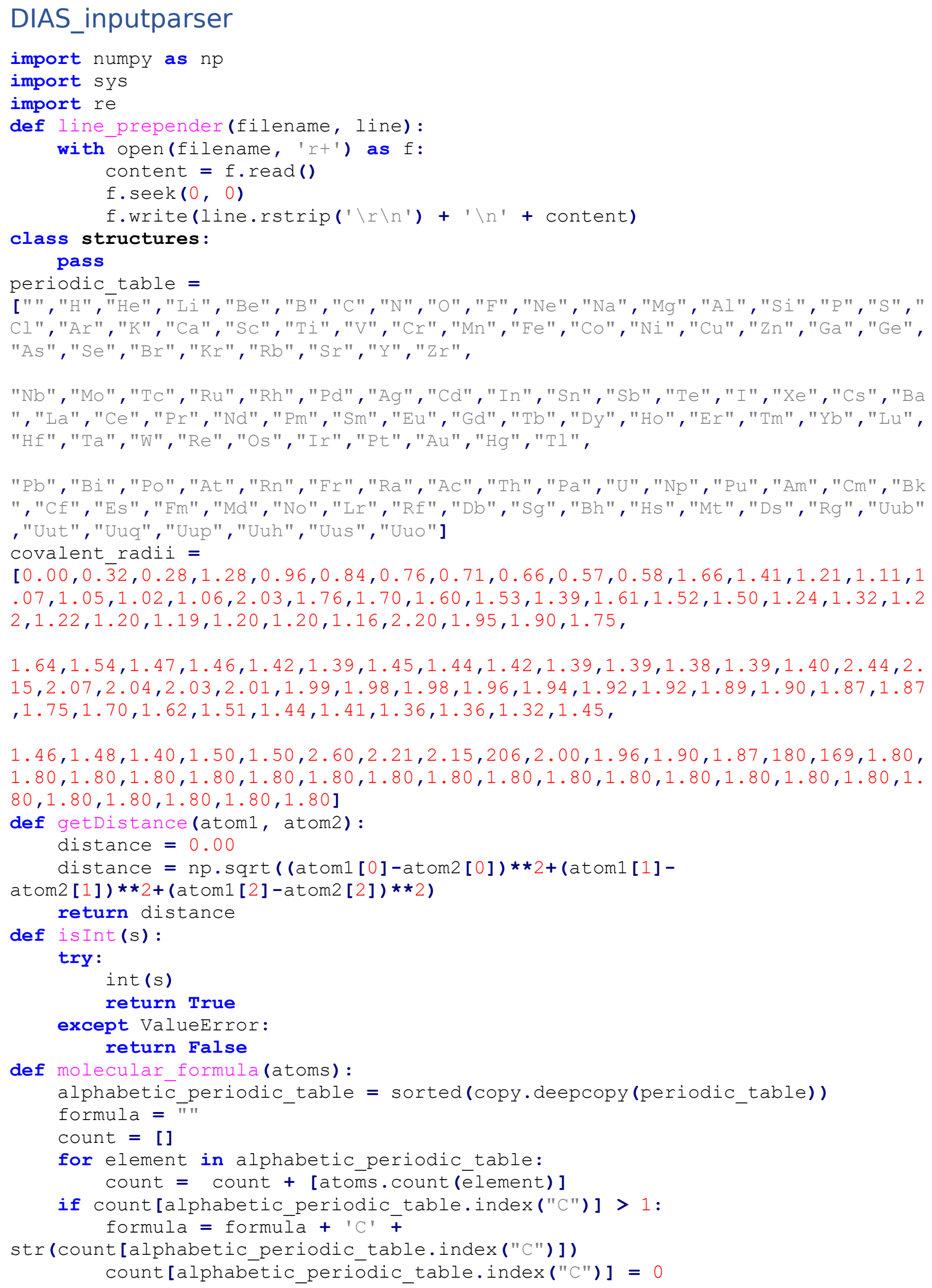




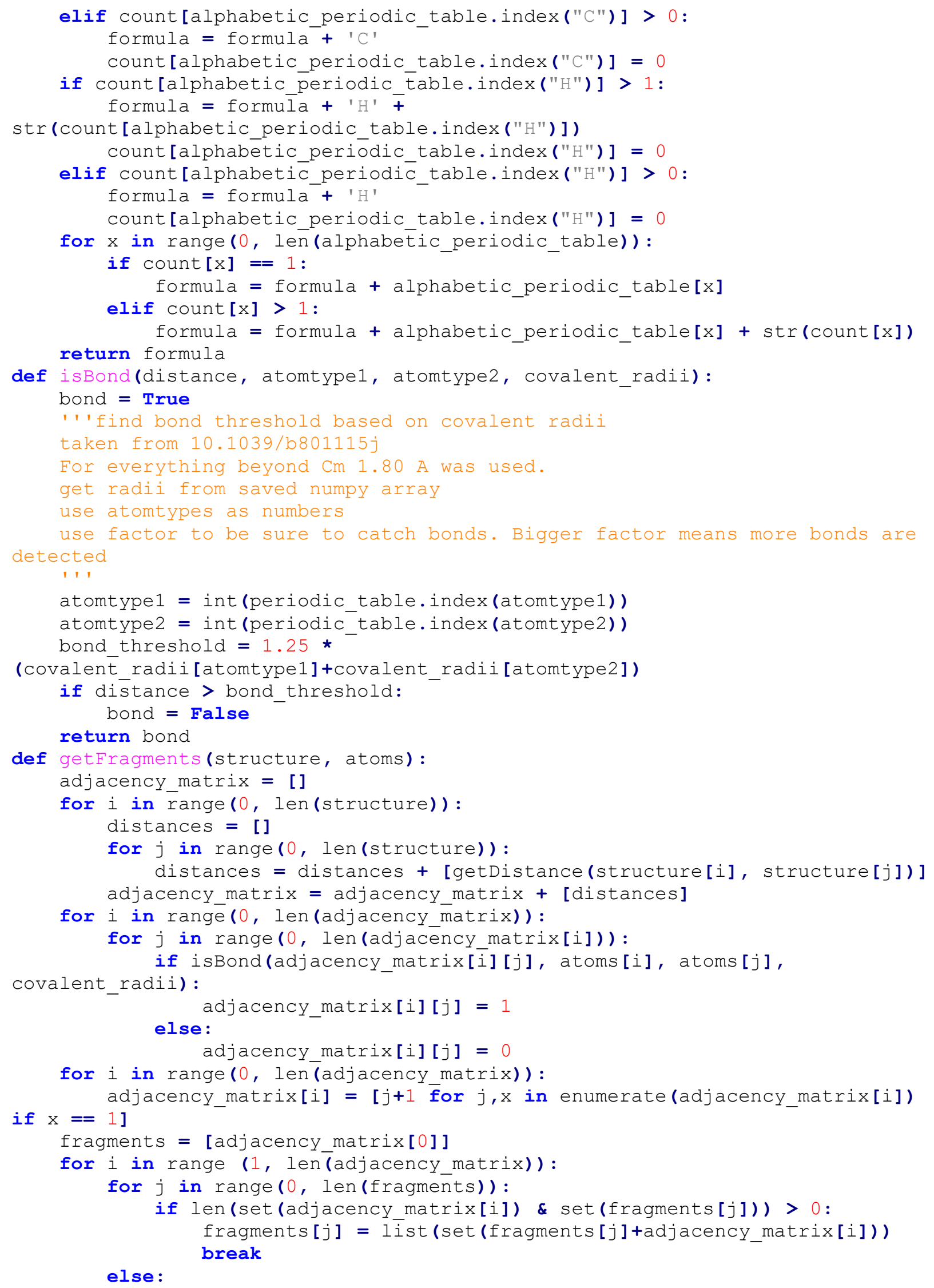




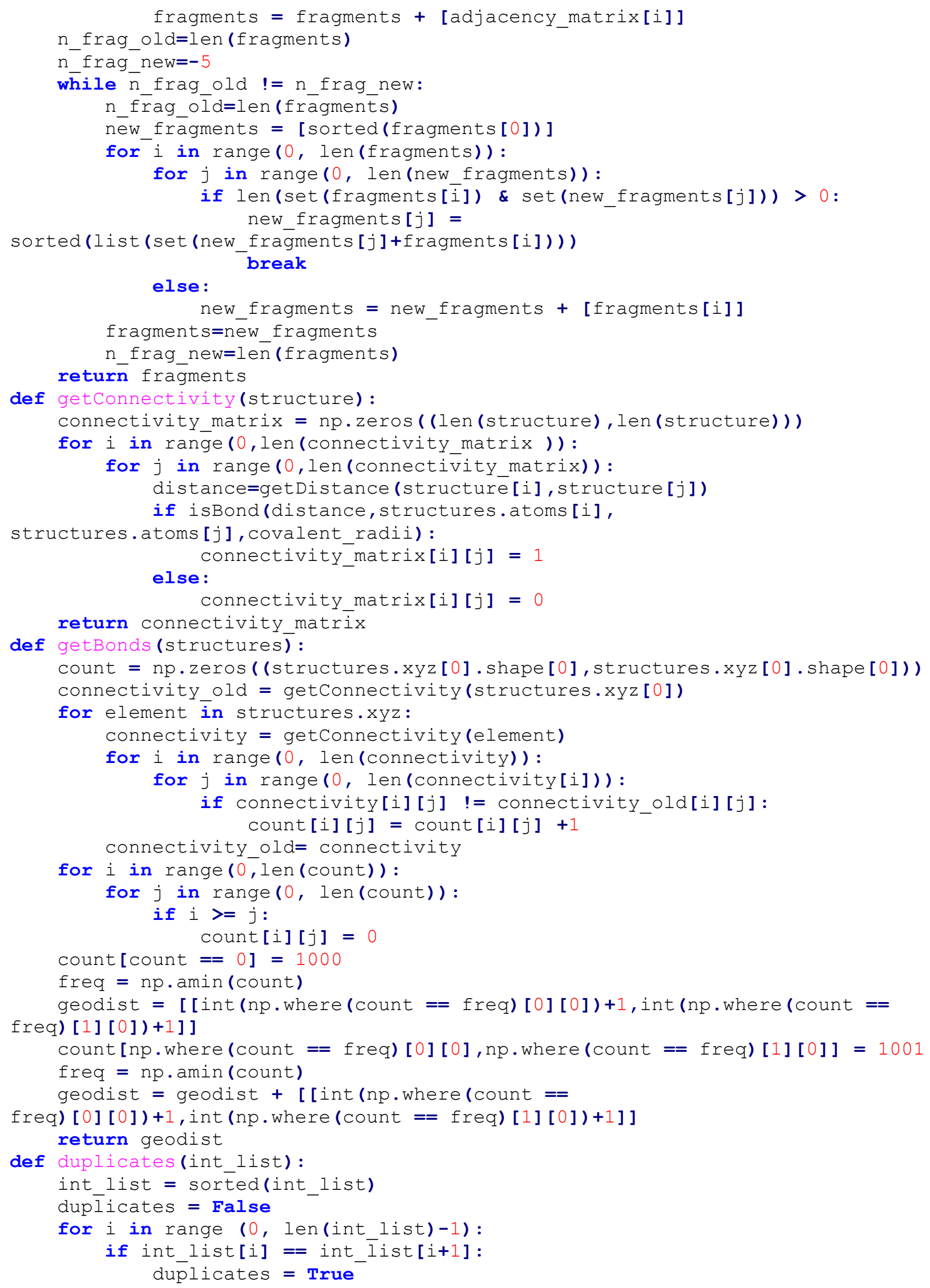


else:

pass

return duplicates

def auto_frag(structures, settings):

list_of fragments $=$ []

for $\bar{i}$ in range (0, len(structures.xyz)):

list of fragments $=$ list of fragments +

[getFragments (structures.xyz[i], structures.atoms)]

$n$ fragments $=[$ ]

for $i$ in range ( 0 , len(list_of fragments)):

$n$ fragments $=$ n_fragments + [len(list_of_fragments[i])]

twofrag = False

morefrag $=$ False

for element in $\mathrm{n}$ fragments:

if element $==2$ :

twofrag $=$ True

break

if element > 2 :

morefrag $=$ True

if twofrag:

indices $=$ [ $i$ for $i, x$ in enumerate (n fragments) if $x==2$ ]

indices ]

counts = [list_of_fragments. count (list_of_fragments[x]) for $x$ in

fragments $=$ list of fragments [indices[counts.index(max(counts))]

settings.fraglatoms $=$ np.sort (fragments [0])

settings.frag2atoms $=$ np.sort(fragments [1])

elif morefrag:

$\mathrm{n}$ fragments $=[\mathrm{x}$ for $\mathrm{x}$ in $\mathrm{n}$ fragments if $\mathrm{x}>2$ ]

iñdices $=$ [ $i$ for $i, x$ in enümerate ( $n$ fragments) if $x==$

min (n_fragments)]

indices] counts = [list_of_fragments. count(list_of_fragments[x]) for $x$ in

fragments $=$ list_of_fragments[indices[counts.index(max(counts))] ]

settings.fraglatoms $=n p \cdot \operatorname{sort}($ fragments $[0])$

settings.frag2atoms $=n p \cdot \operatorname{sort}([j$ for $i$ in fragments [1:] for $j$ in $i])$

line_prepender(settings.logfile, "WARNING: more than two fragments

detected! Check fragments! \n\n")

else:

line prepender(settings.logfile, "CRITICAL ERROR: couldn't determine fragments automatically! \n\n")

sys.exit("CRITICAL ERROR: couldn't determine fragments

automatically! ")

return settings

def get_single(file):

structures. atoms $=[]$

structures. $\mathrm{xyz}=[\mathrm{]}$

structures.title $=$ ['single structure']

with open(file) as input_file:

file_contents = input_file.read()

if "Optimization complete $\bar{d}$." in file_contents:

regex $=r$

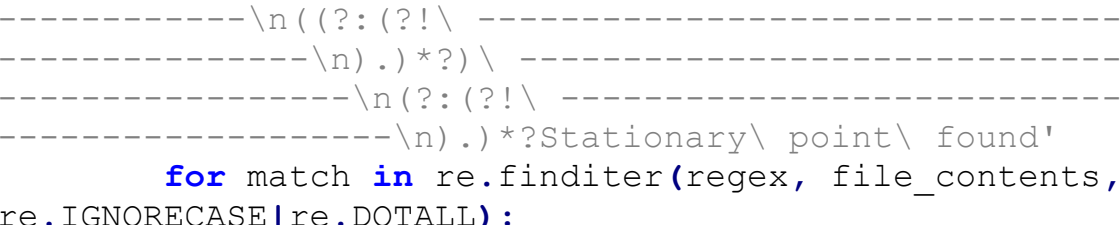


else:

regex $=r^{\prime}$

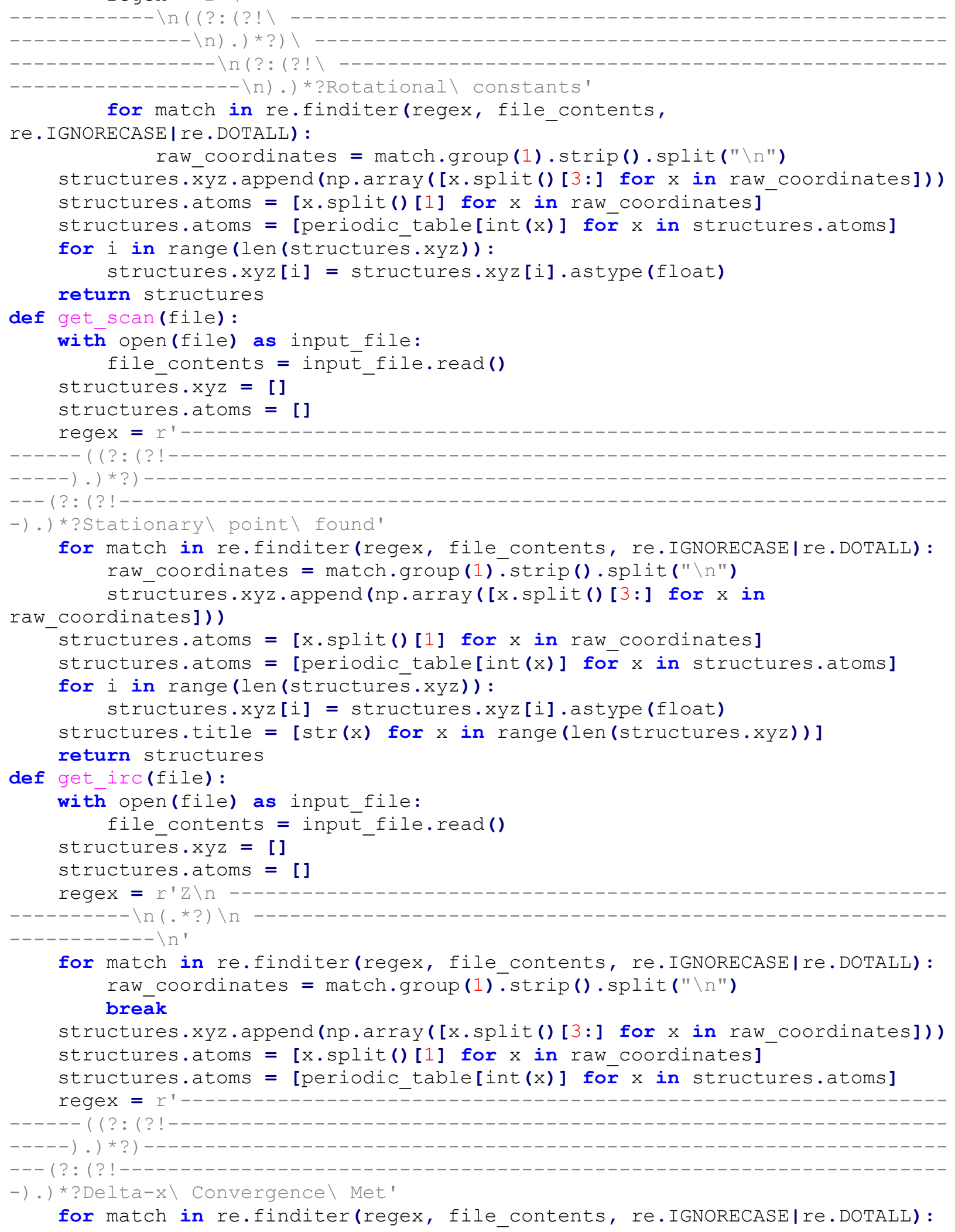




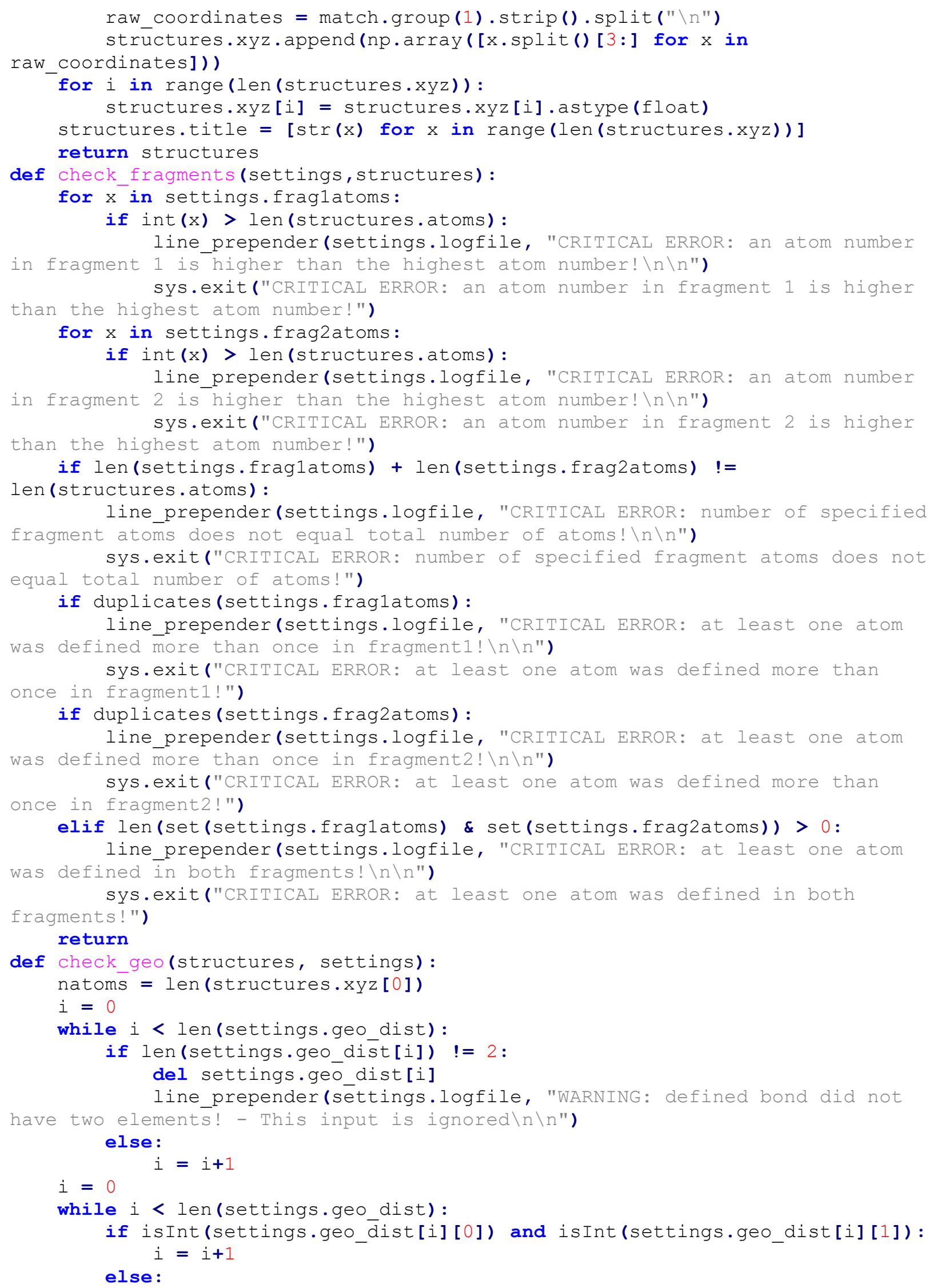


del settings.geo_dist[i]

line prepender(sēttings.logfile, "WARNING: defined bond did have non-integer input! - This input is ignored $\backslash n \backslash n "$ )

if len(settings.geo dist) $==0$ :

settings.geo_dist.append (getBonds (structures))

line_prepender(settings.logfile, "WARNING: no correct definitions of

bonds found! - automatic detection of forming bonds $\backslash n \backslash n "$ )

$$
i=0
$$

while $i<$ len(settings.geo_dist):

if int (settings.geo_dist[i][0]) > natoms or

int(settings.geo_dist[i][1]) > natoms or int(settings.geo_dist[i][0]) < 1 or int (settings.geo_dist[i][1]) $<1$ :

del settings.geo_dist[i]

line_prepender(settings.logfile, "WARNING: bond definition: atom

number out of range! - This input is ignored $\backslash n \backslash n "$ )

else:

$i=i+1$

$i=0$

while $i<$ len(settings.geo_ang):

if len(settings.geo ang $\overline{\text { [i] }}$ ) $\quad !=3$ :

del settings.geo_ang[i]

line_prepender(sēttings.logfile, "WARNING: defined angle did not

have three elements! - This input is ignored $\backslash n \backslash n "$ )

else:

$$
i=i+1
$$

$i=0$

while $i<$ len(settings.geo_ang):

if isInt(settings.geo ang[i][0]) and isInt(settings.geo_ang[i][1])

and isInt (settings.geo ang[i][2]):

else:

$i=i+1$

del settings.geo_ang[i]

line_prepender(sēttings.logfile, "WARNING: defined angle did have

non-integer input! - This input is ignored $\backslash n \backslash n "$ )

$i=0$

while $i<$ len(settings.geo_ang):

if int(settings.geo ang [i][0]) > natoms or

int (settings.geo_ang[i][1]) $>$ natoms or int(settings.geo_ang[i][2]) > natoms

or int(settings.geo_ang[i][0]) < 1 or int(settings.geo_ang[i][1]) < 1 or

int (settings.geo_ang [i][2]) $<1$ :

del settings.geo ang[i]

line_prepender(sēttings.logfile, "WARNING: angle definition: atom

number out of range! - This input is ignored $\backslash n \backslash n "$ )

else:

$i=i+1$

$i=0$

while $i<$ len(settings.geo_dih):

if len(settings.geo_dih[i]) != 4 :

del settings.geo_dih[i]

line_prepender(sēttings.logfile, "WARNING: defined dihedral angle

did not have three elements! - This input is ignored $\backslash n \backslash n "$ )

else:

$i=i+1$

$i=0$

while $i<$ len(settings.geo_dih):

if isInt(settings.geo dih[i][0]) and isInt(settings.geo dih[i][1])

and isInt(settings.geo_dih[i][2]) and isInt(settings.geo_dih[i][3]): 


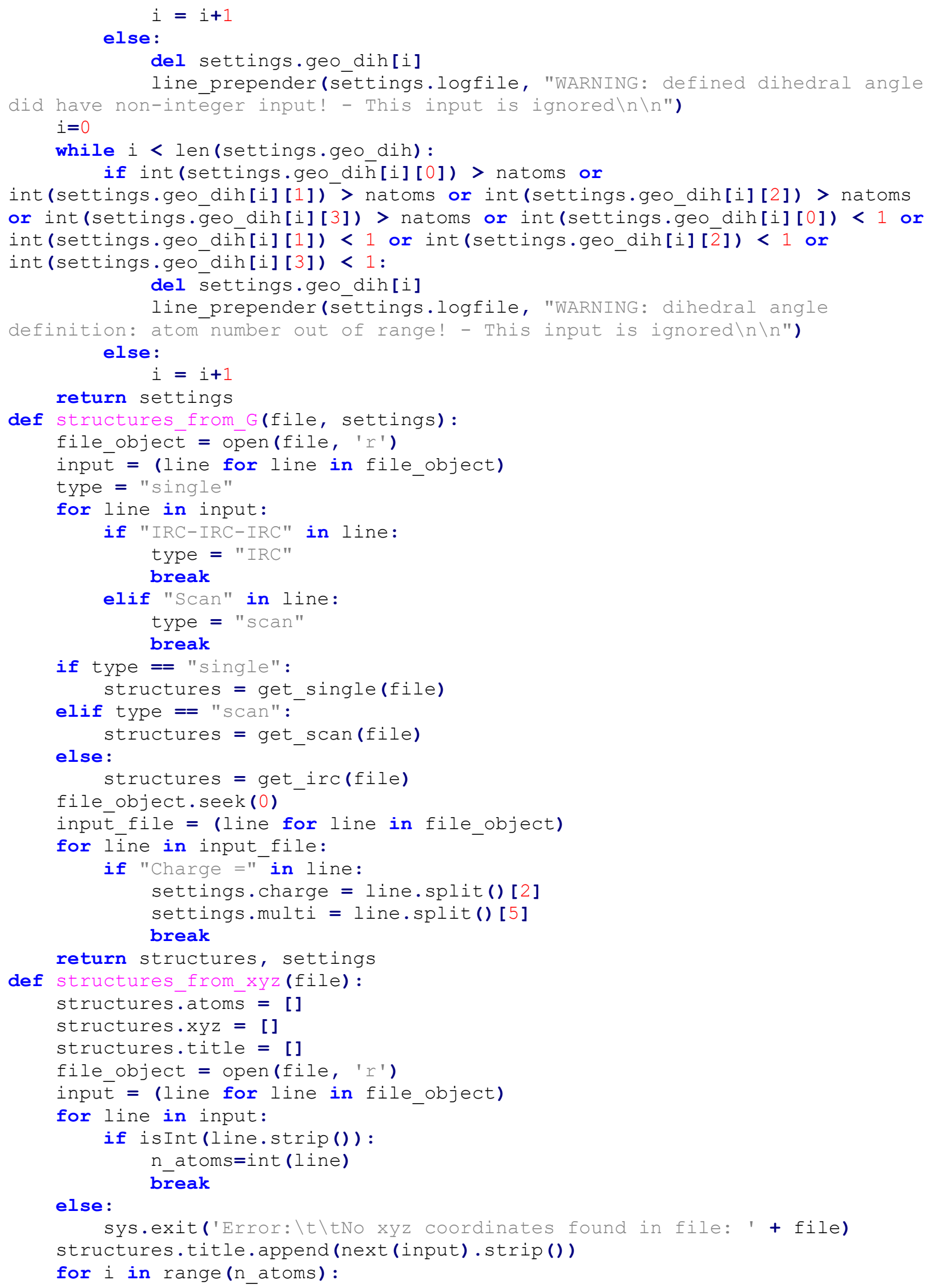




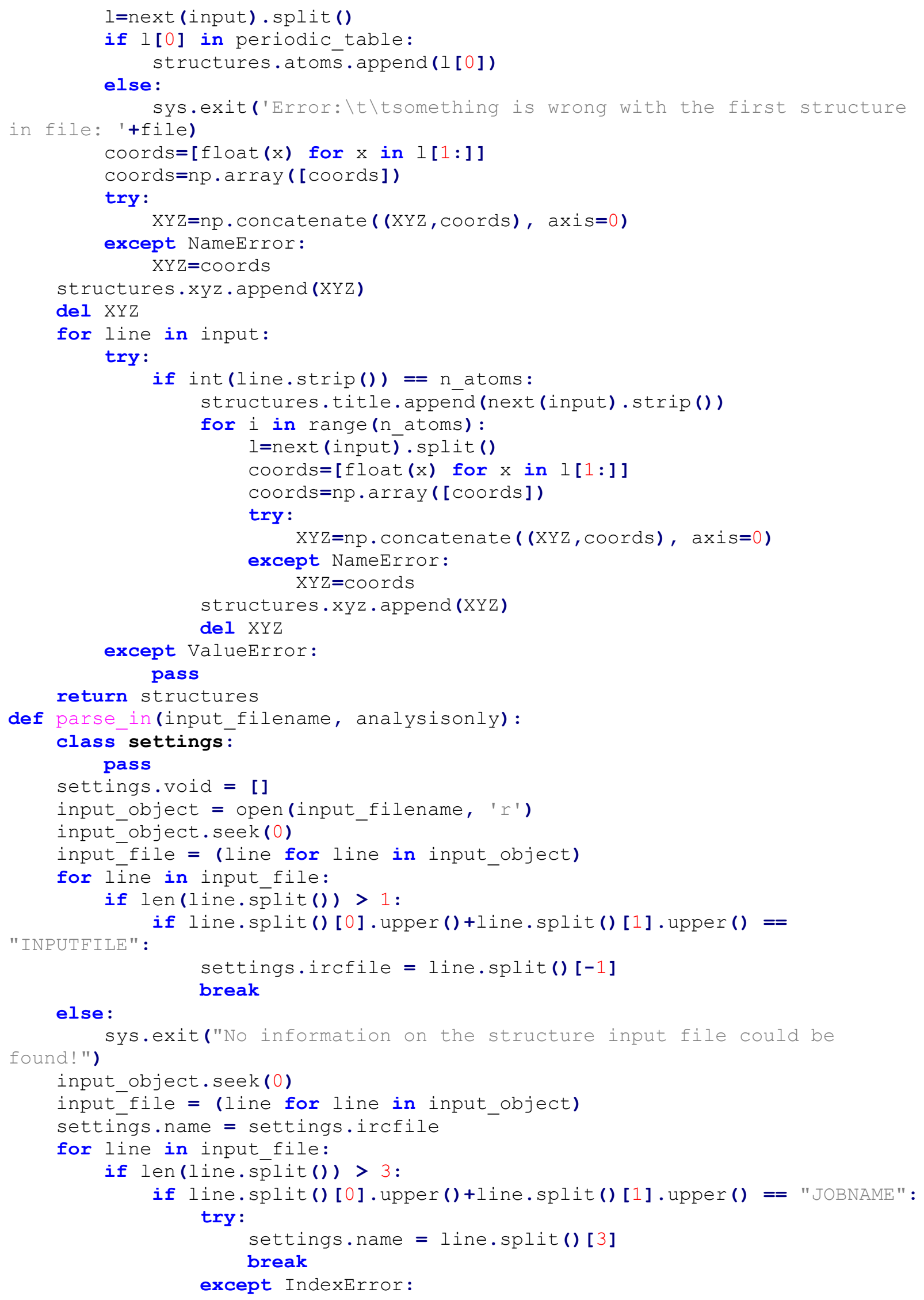




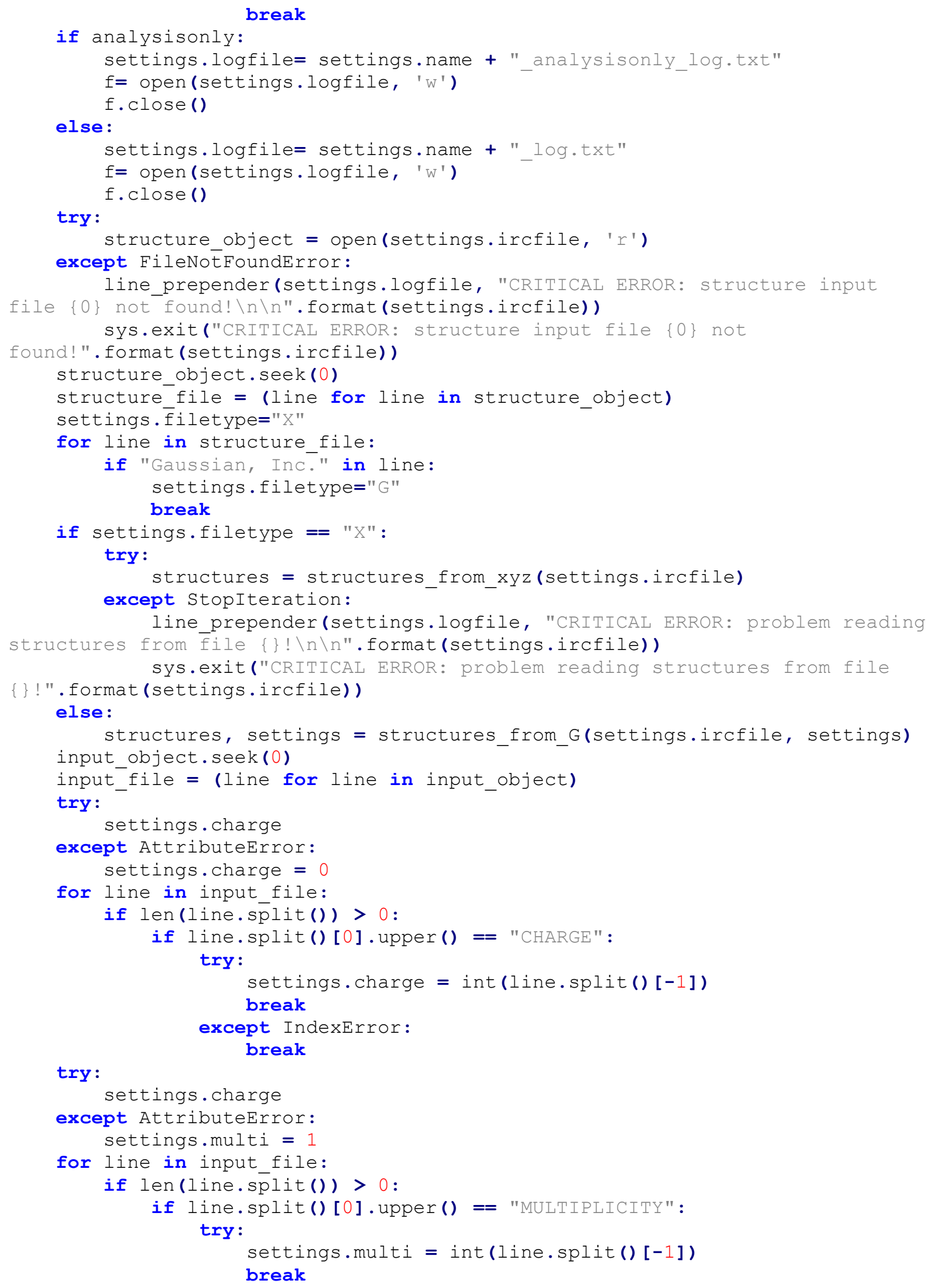




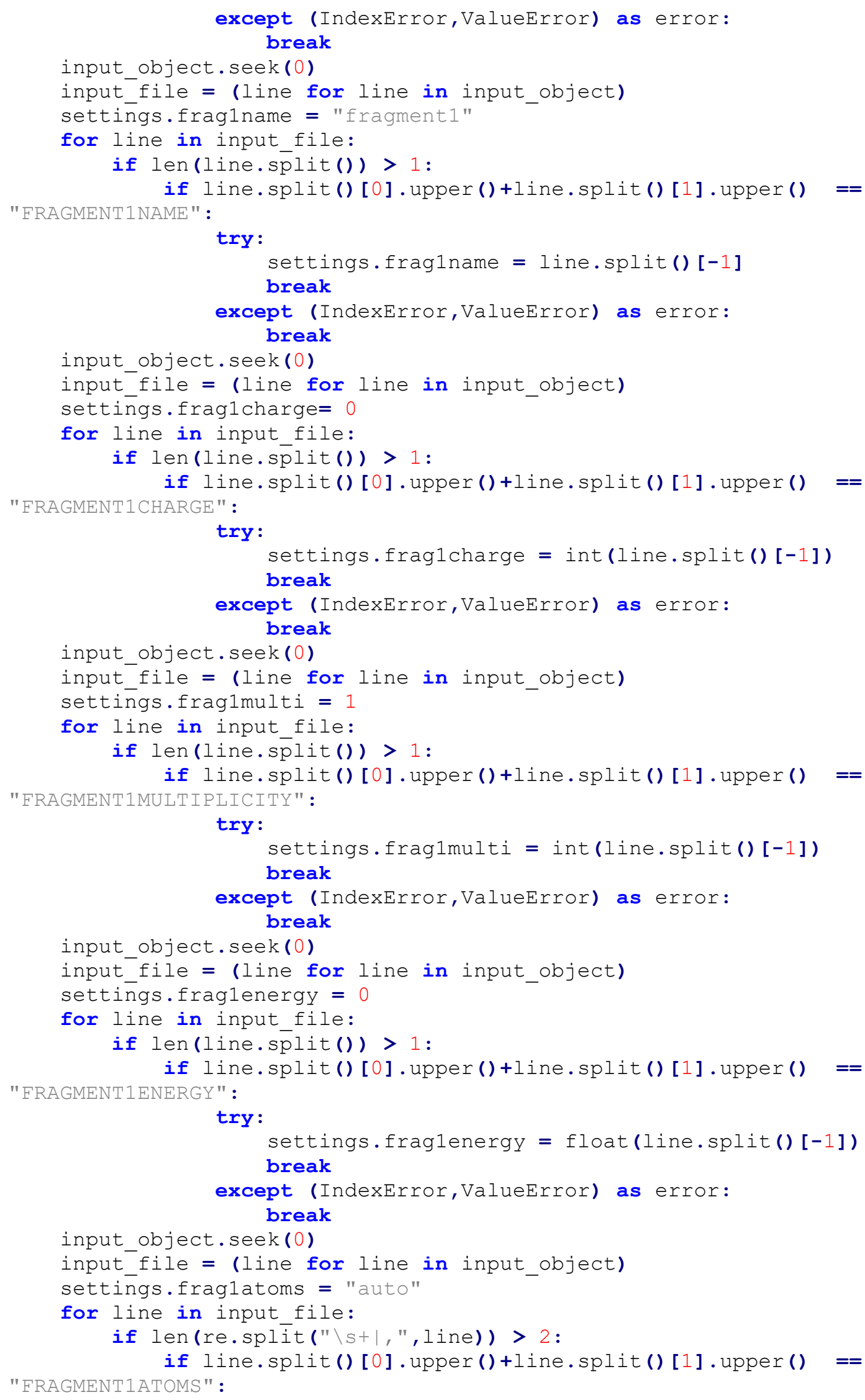




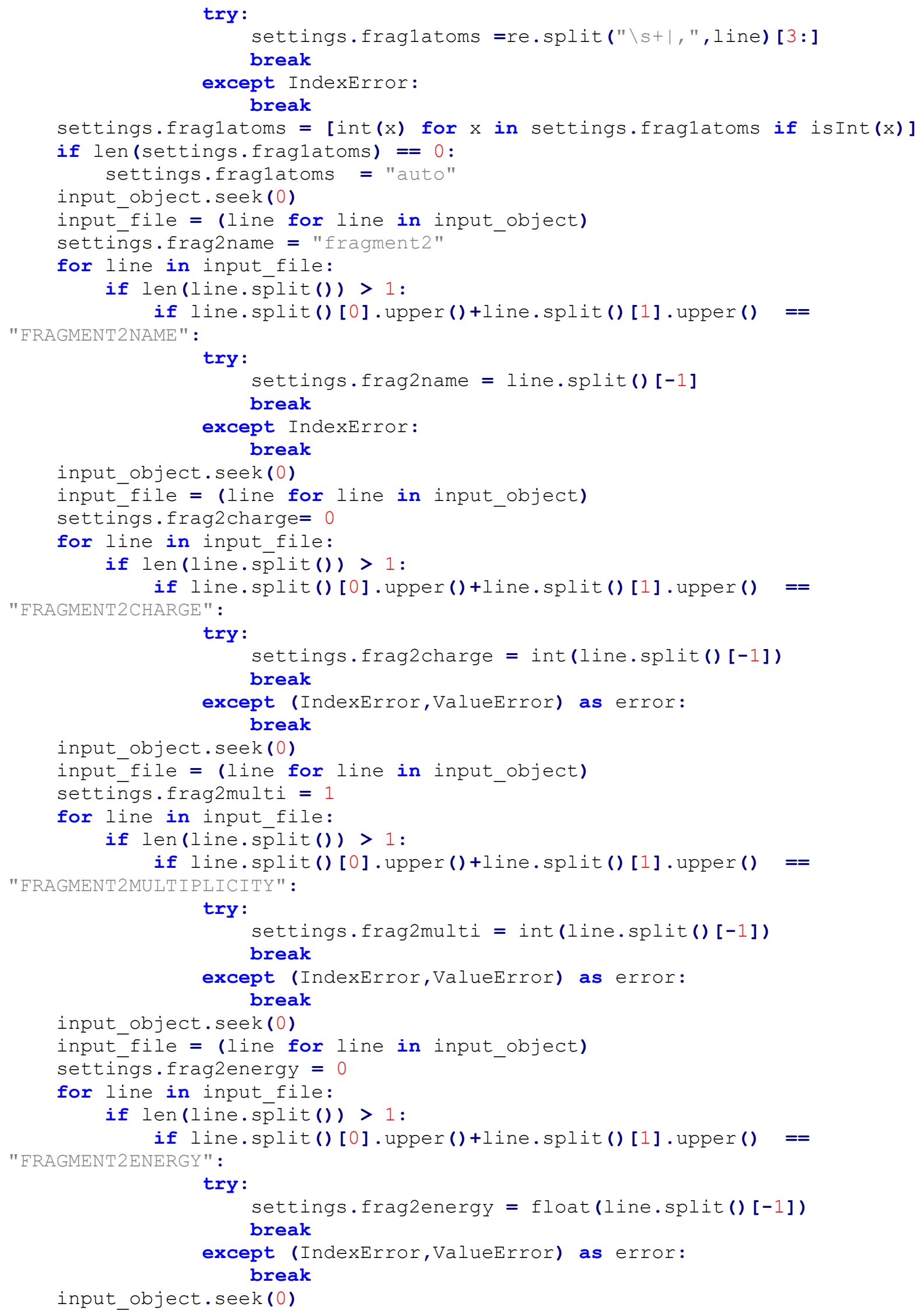




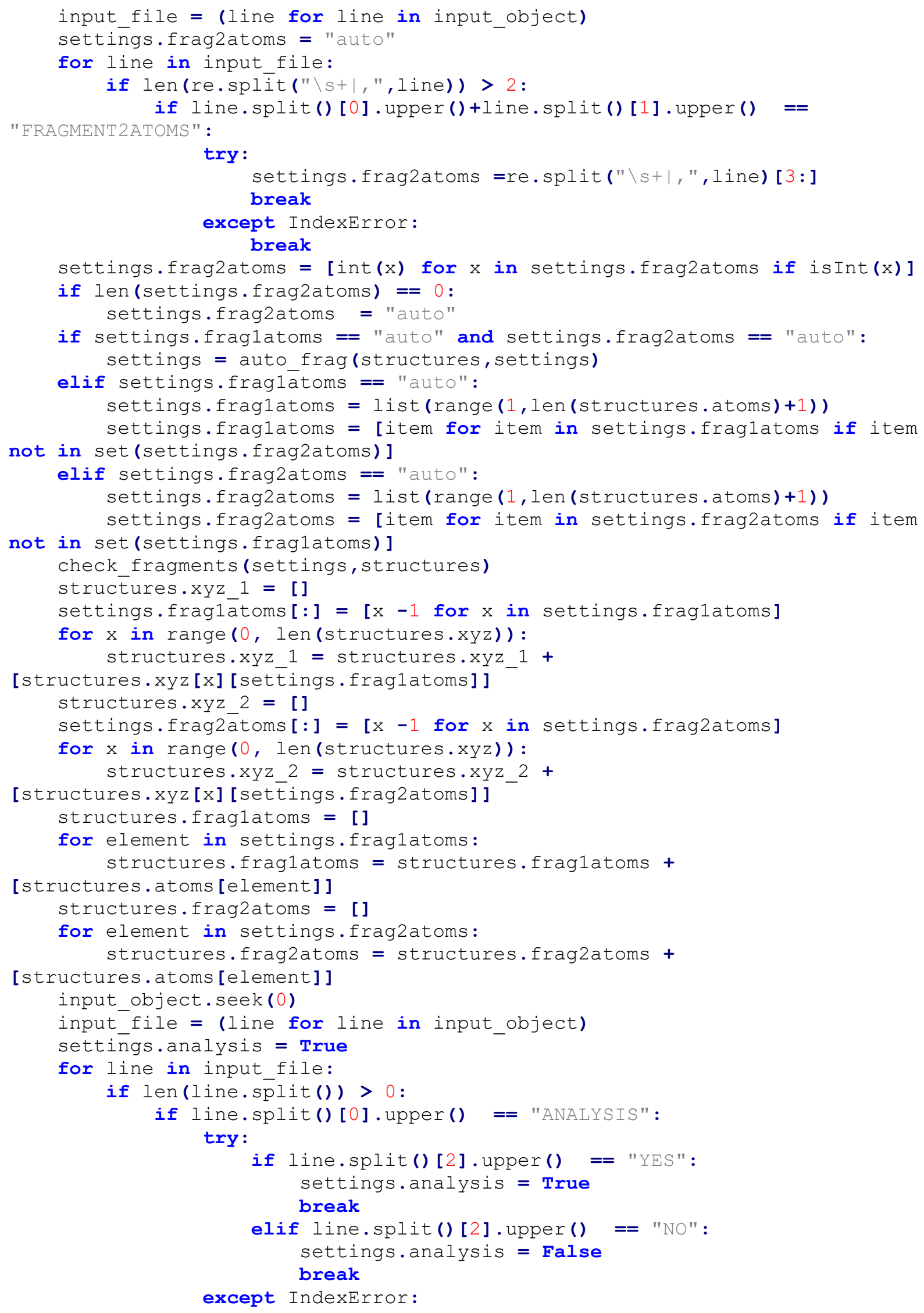




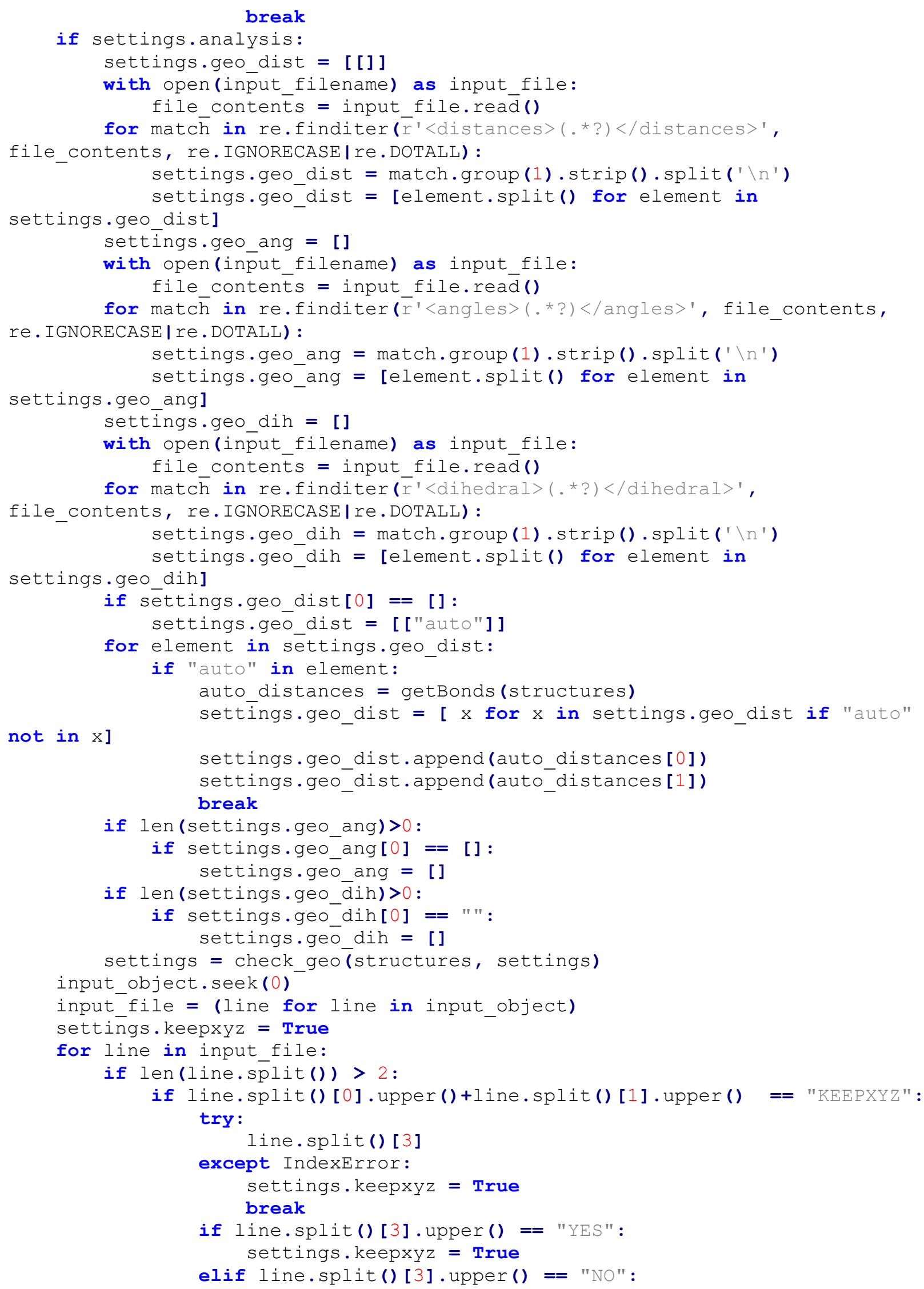




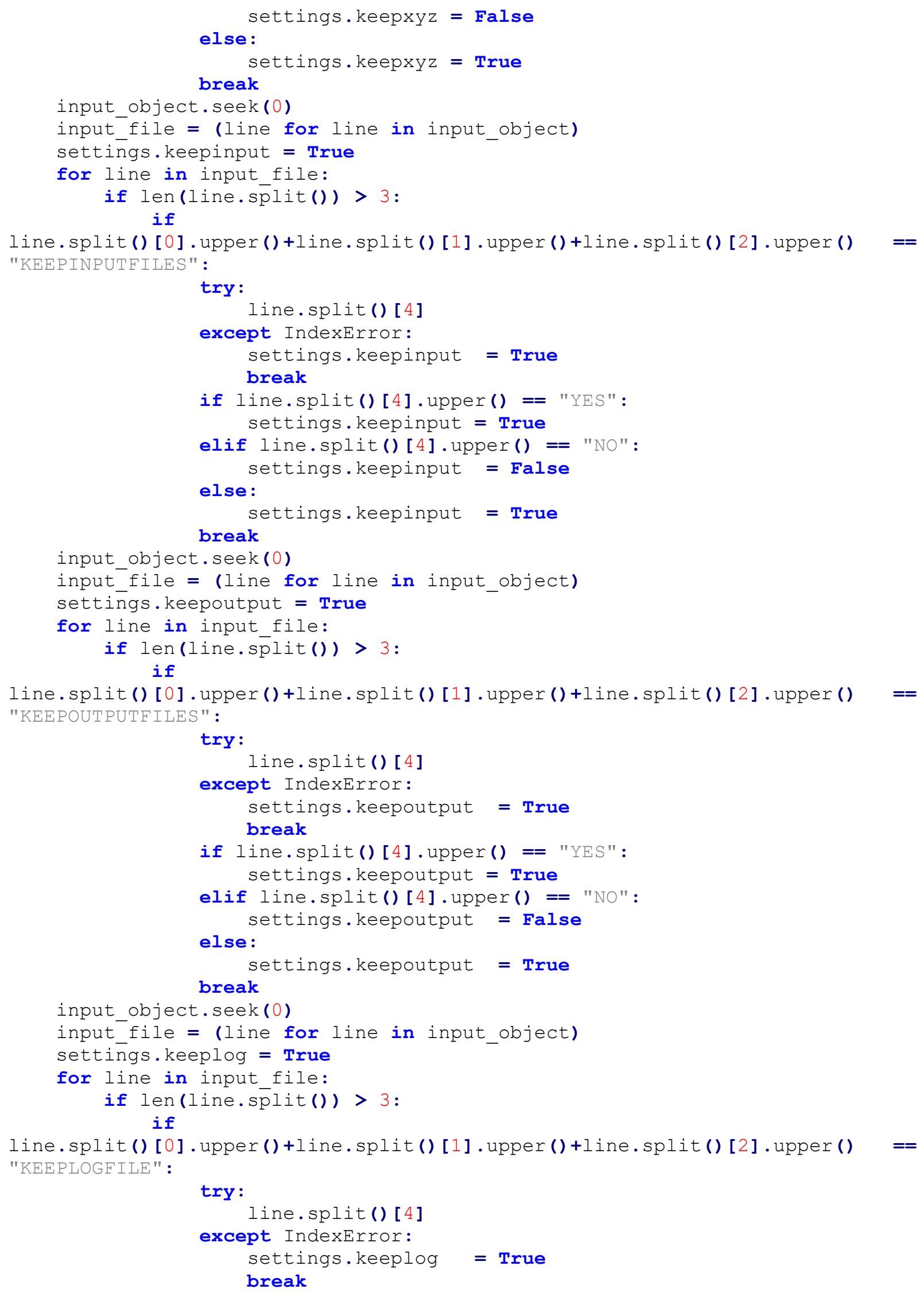




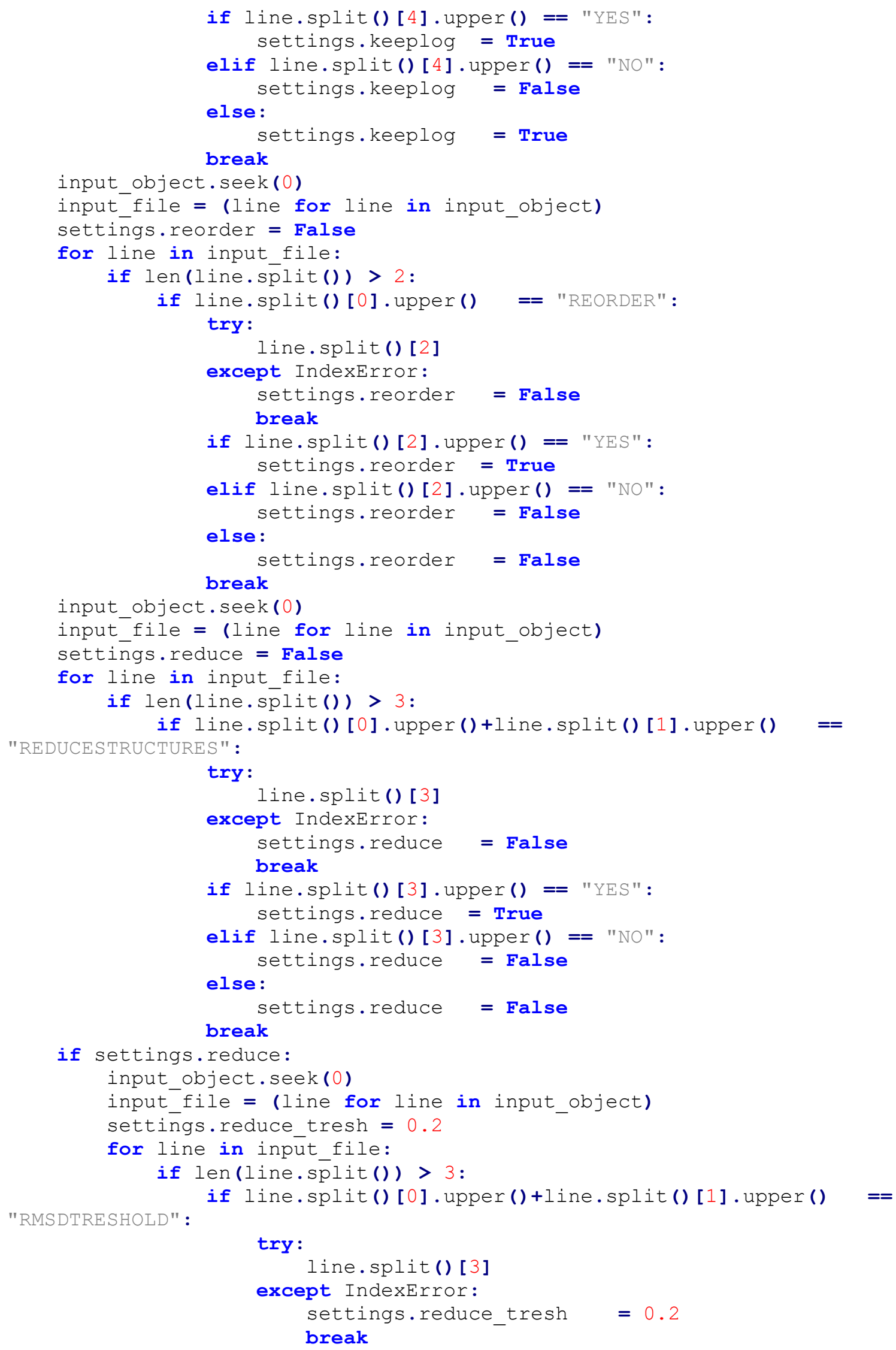




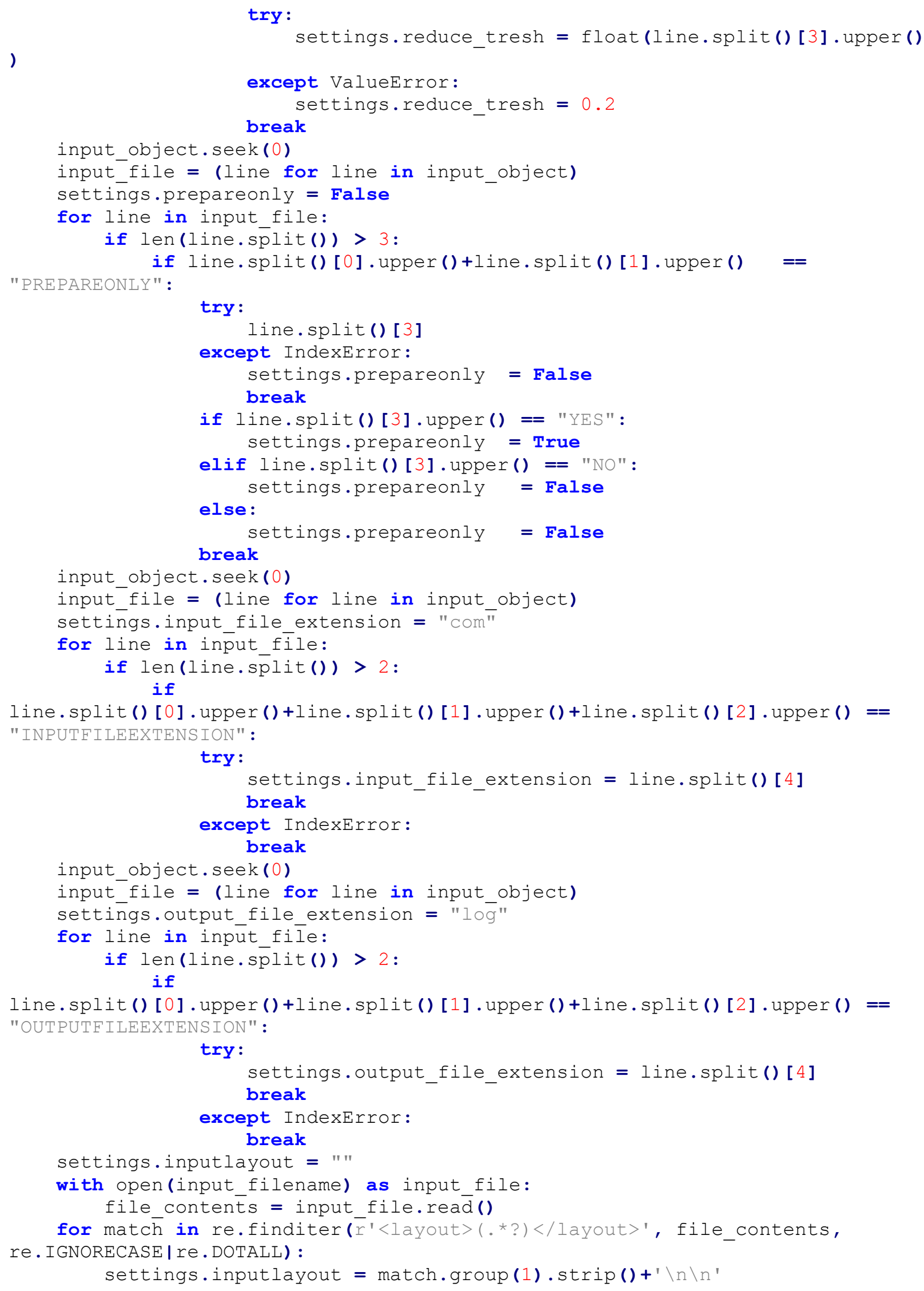




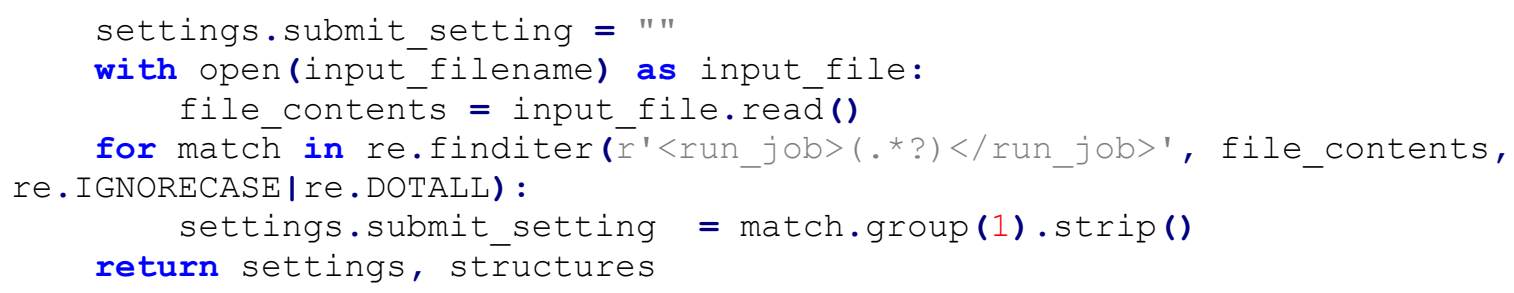




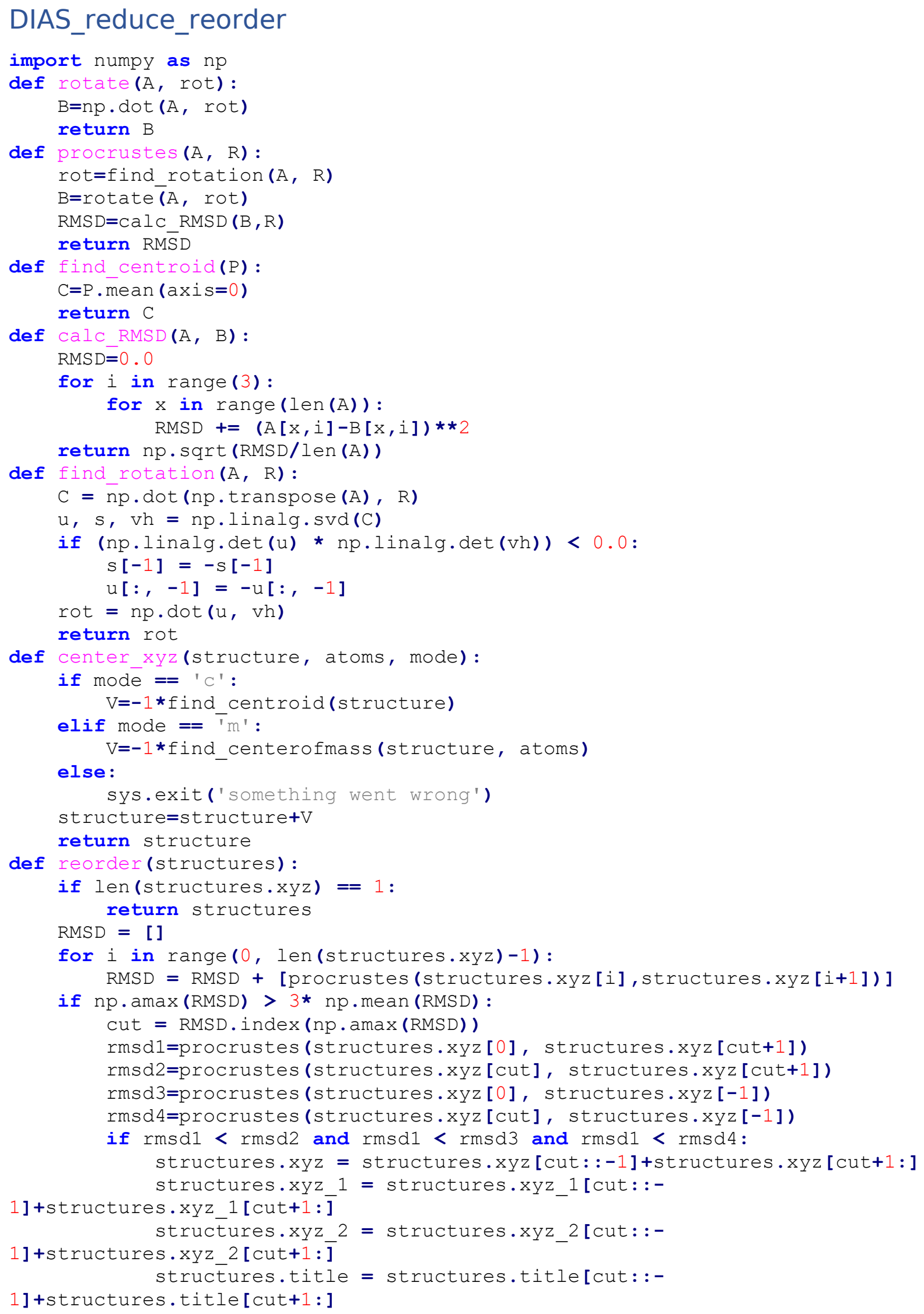




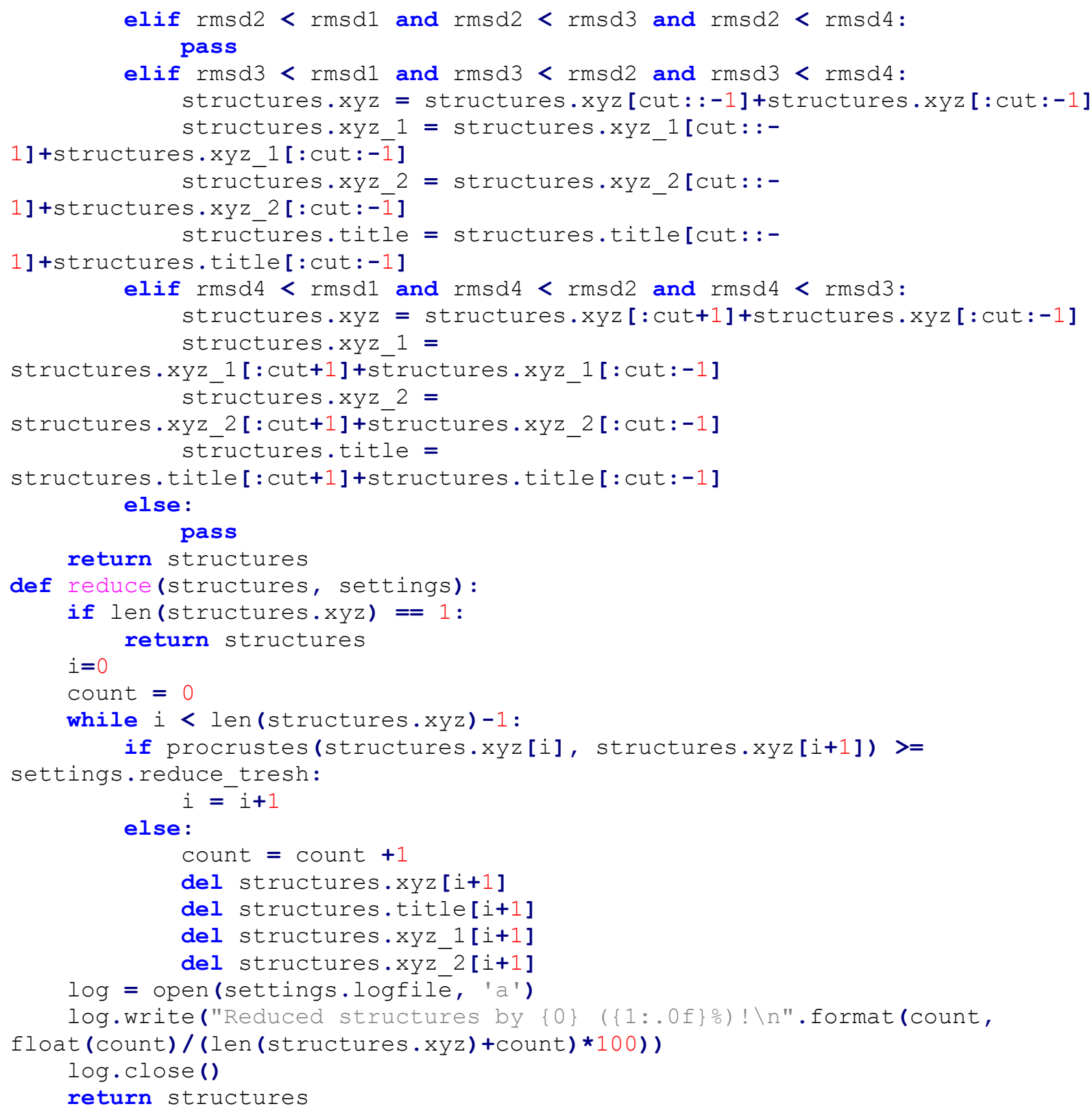




\section{DIAS_analysis}

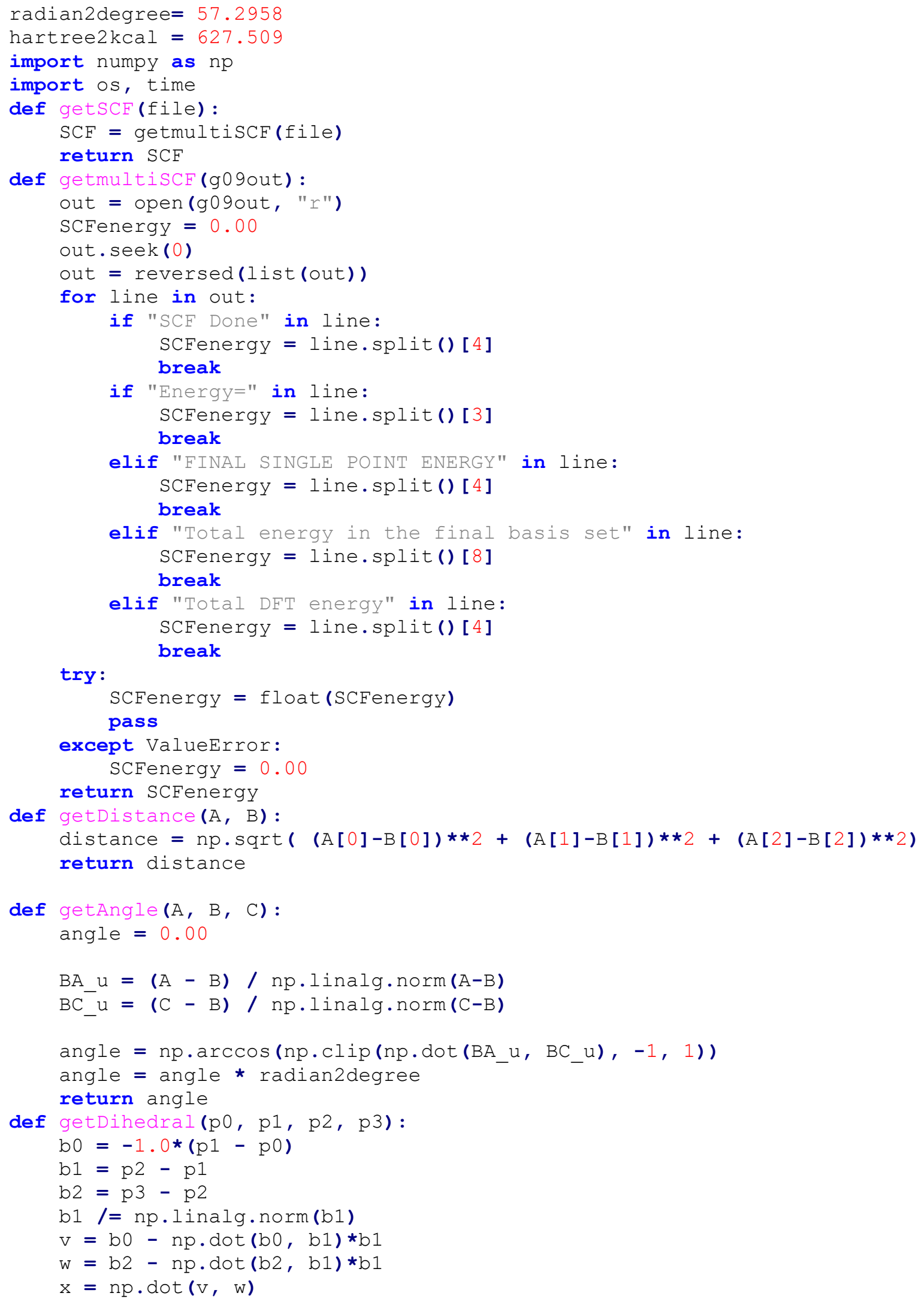




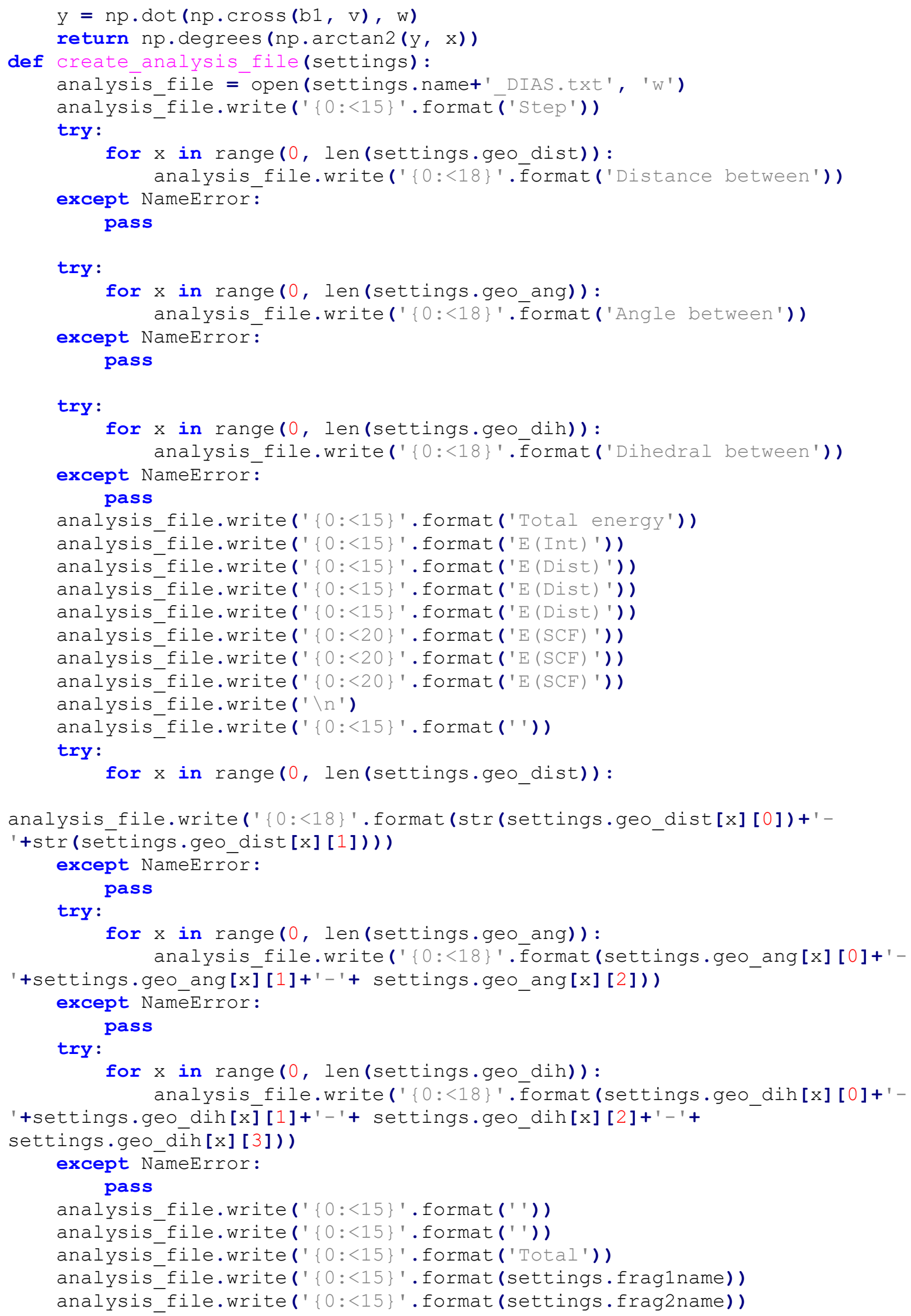




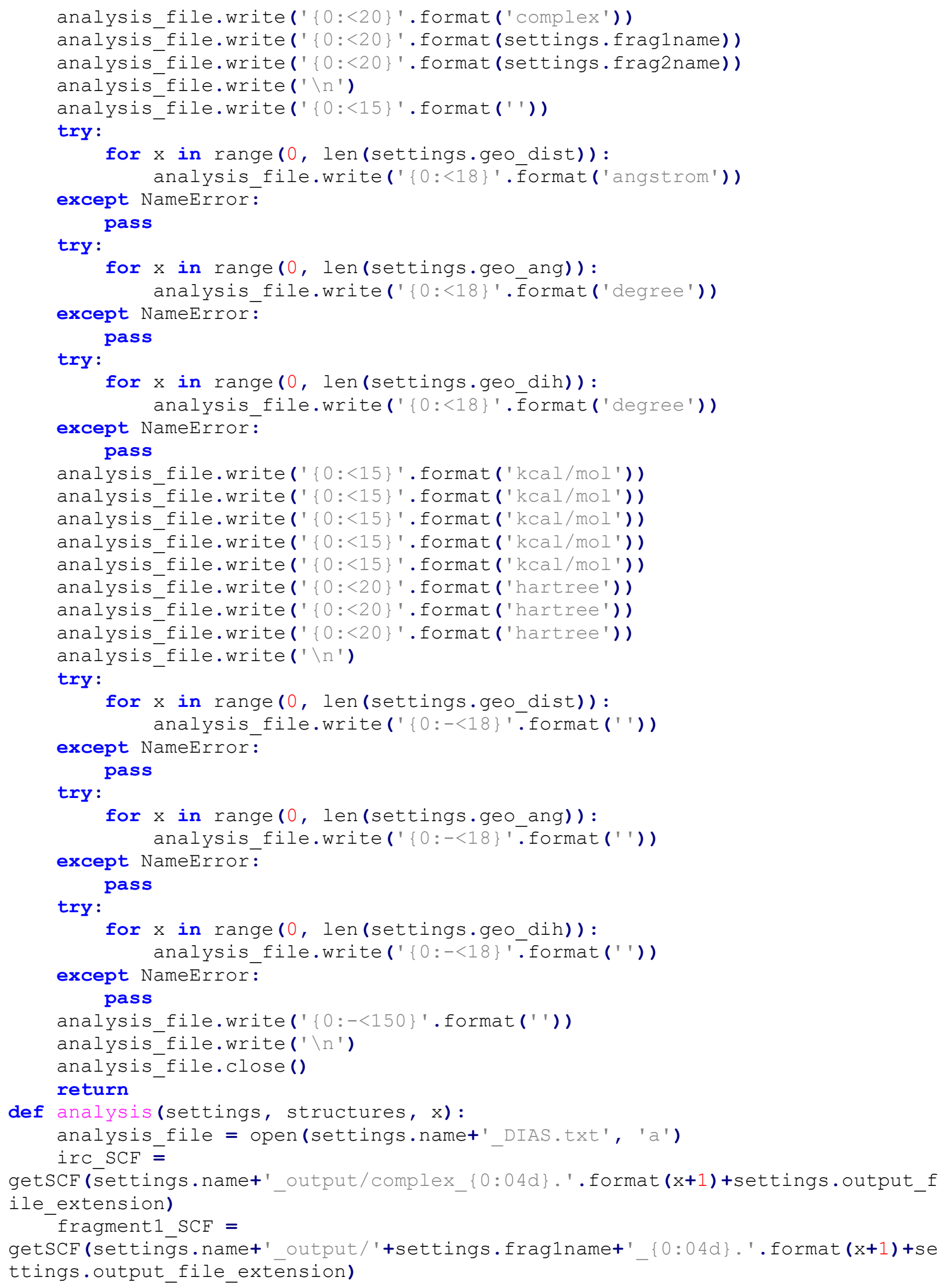




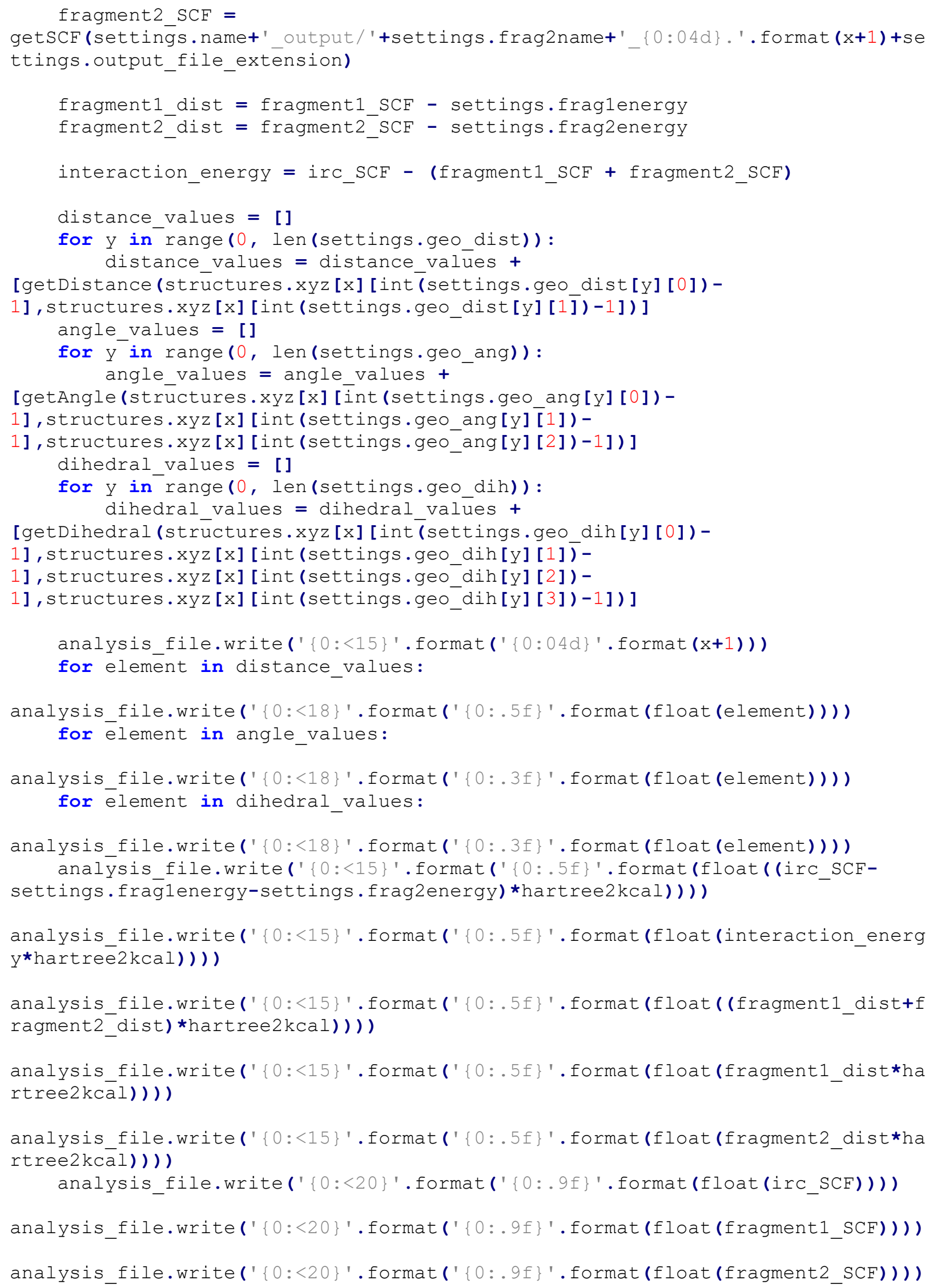




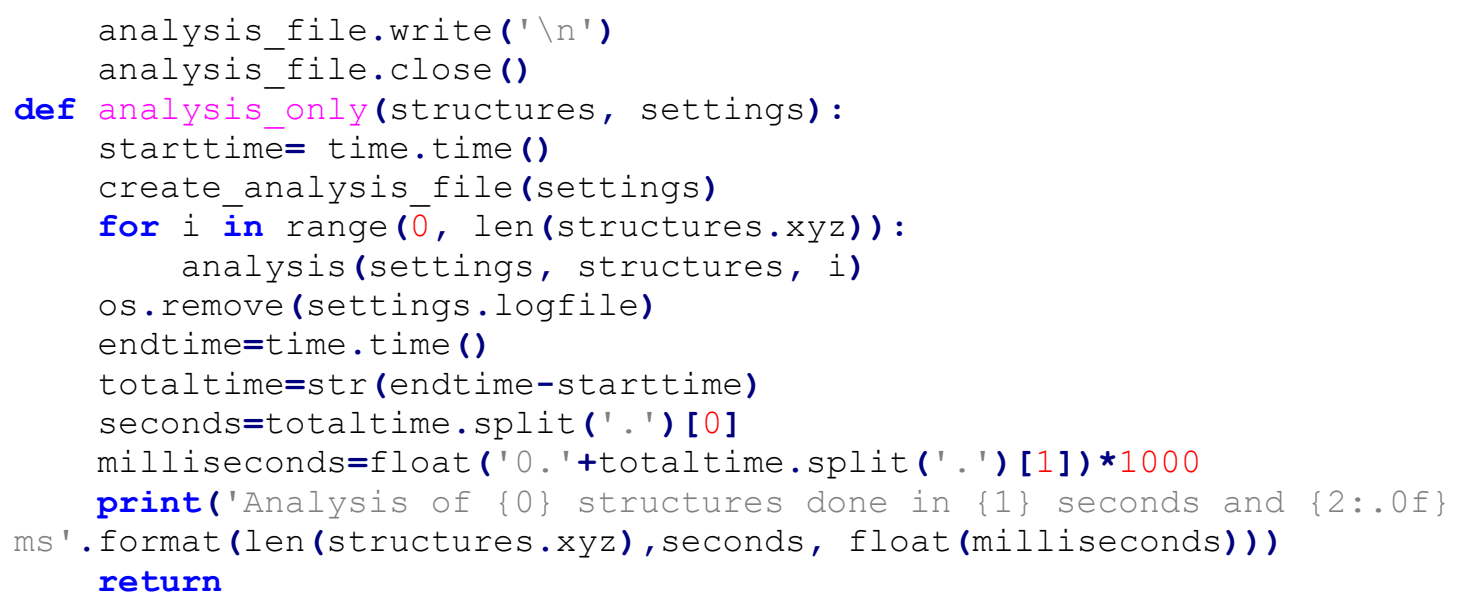




\section{Settings input file}

\section{The settings input file controls the actions taken by autoDIAS. In the following an example showing all available settings is presented. Settings are not case sensitive.}

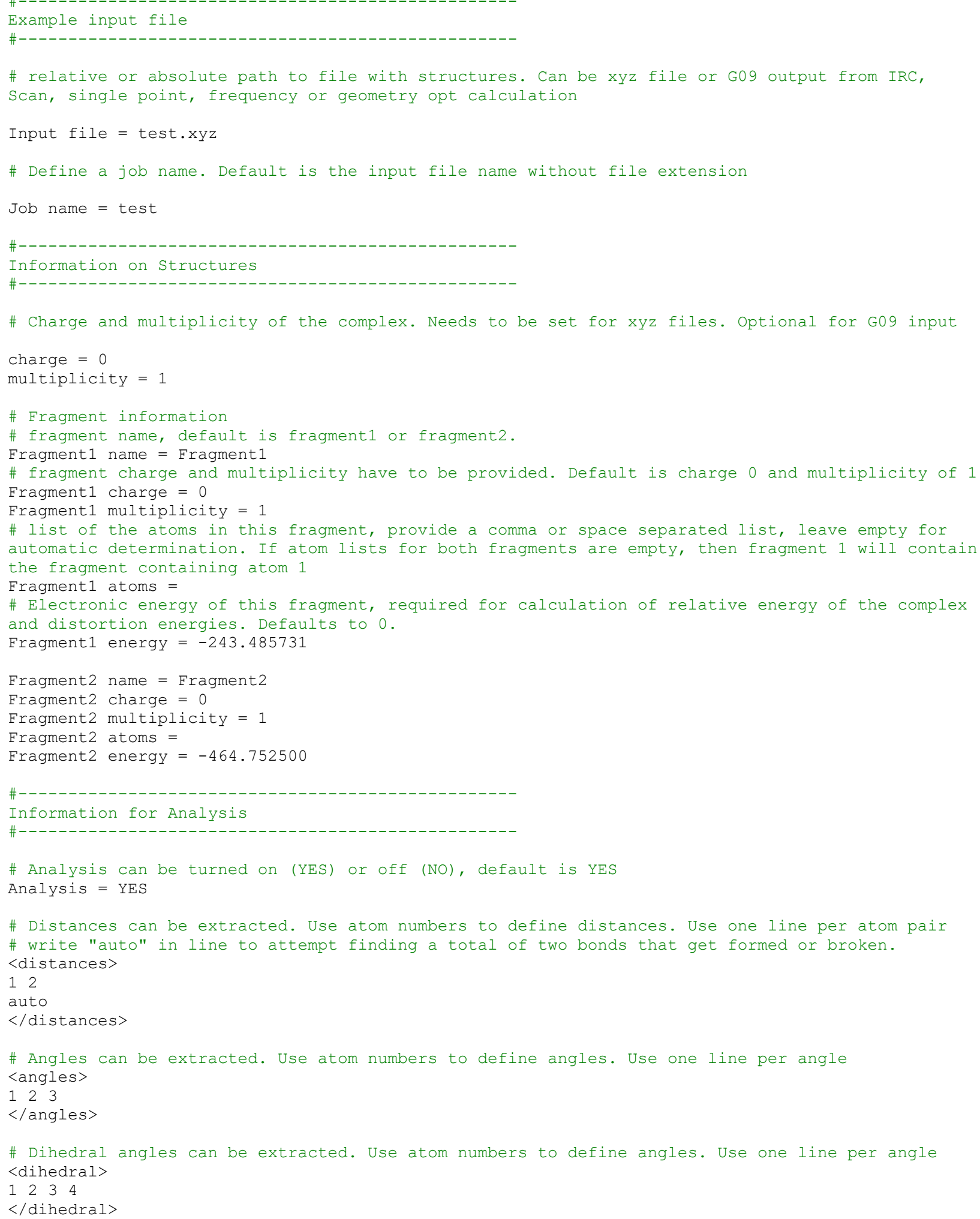




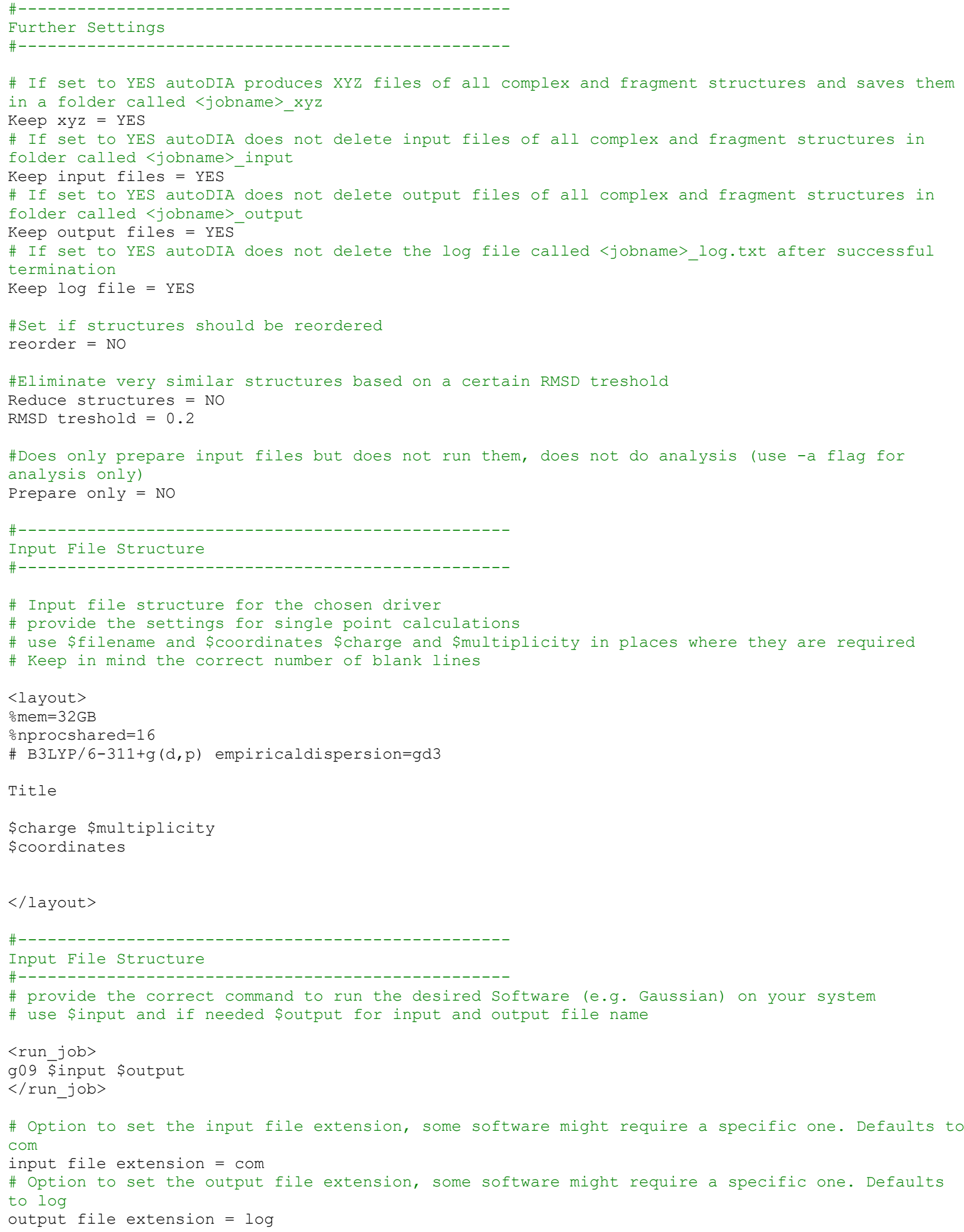




\section{Log file}

\section{The log file contains information about the performed DIAS analysis. An example is shown here.}

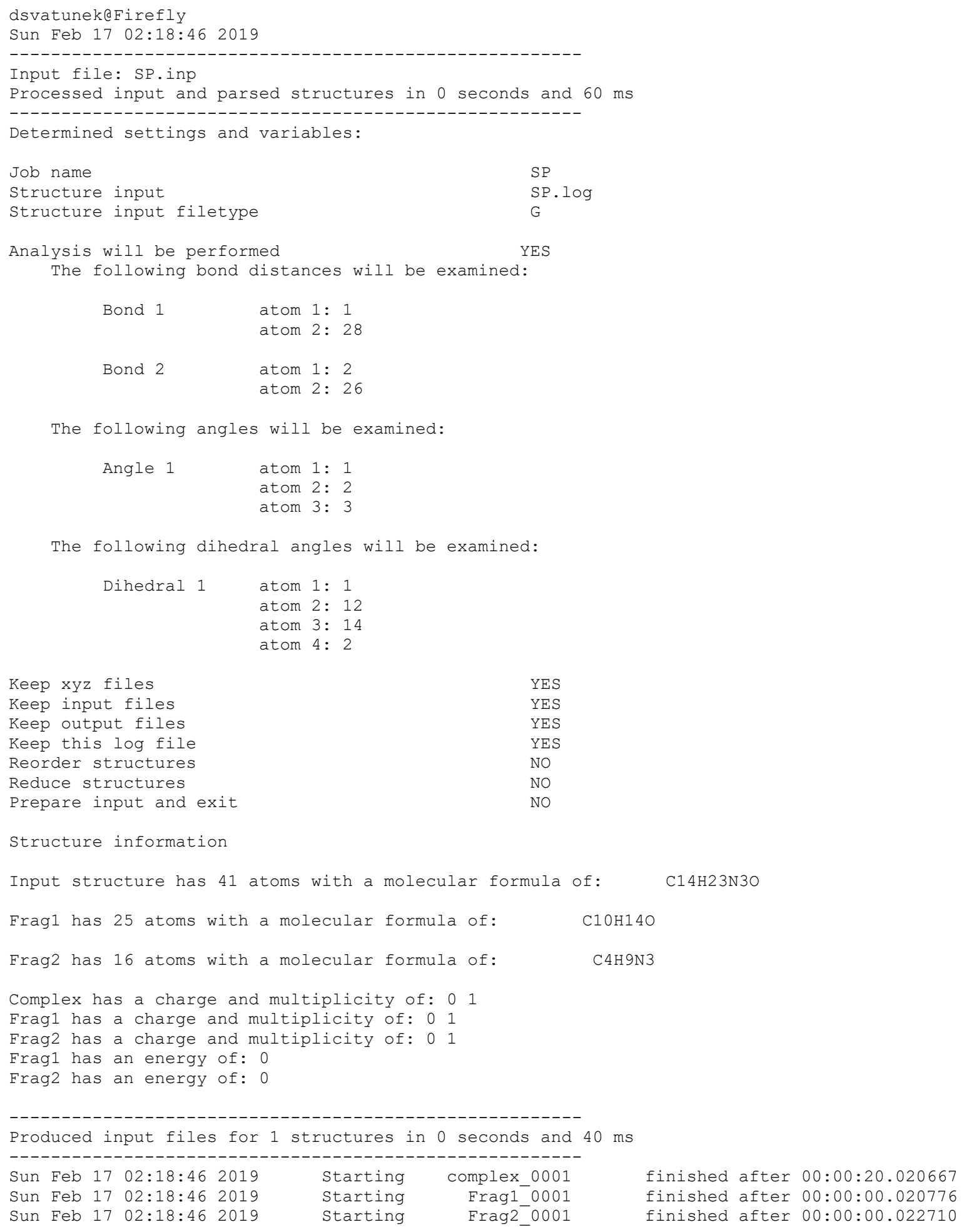




\section{DIAS file}

The DIAS file named <jobname>_DIAS.txt contains all extracted information. The layout follows point numbers, geometric parameters, calculate $\bar{d}$ energies and extracted energies. The exact layout depends on the number of geometric parameters. An example is shown here.

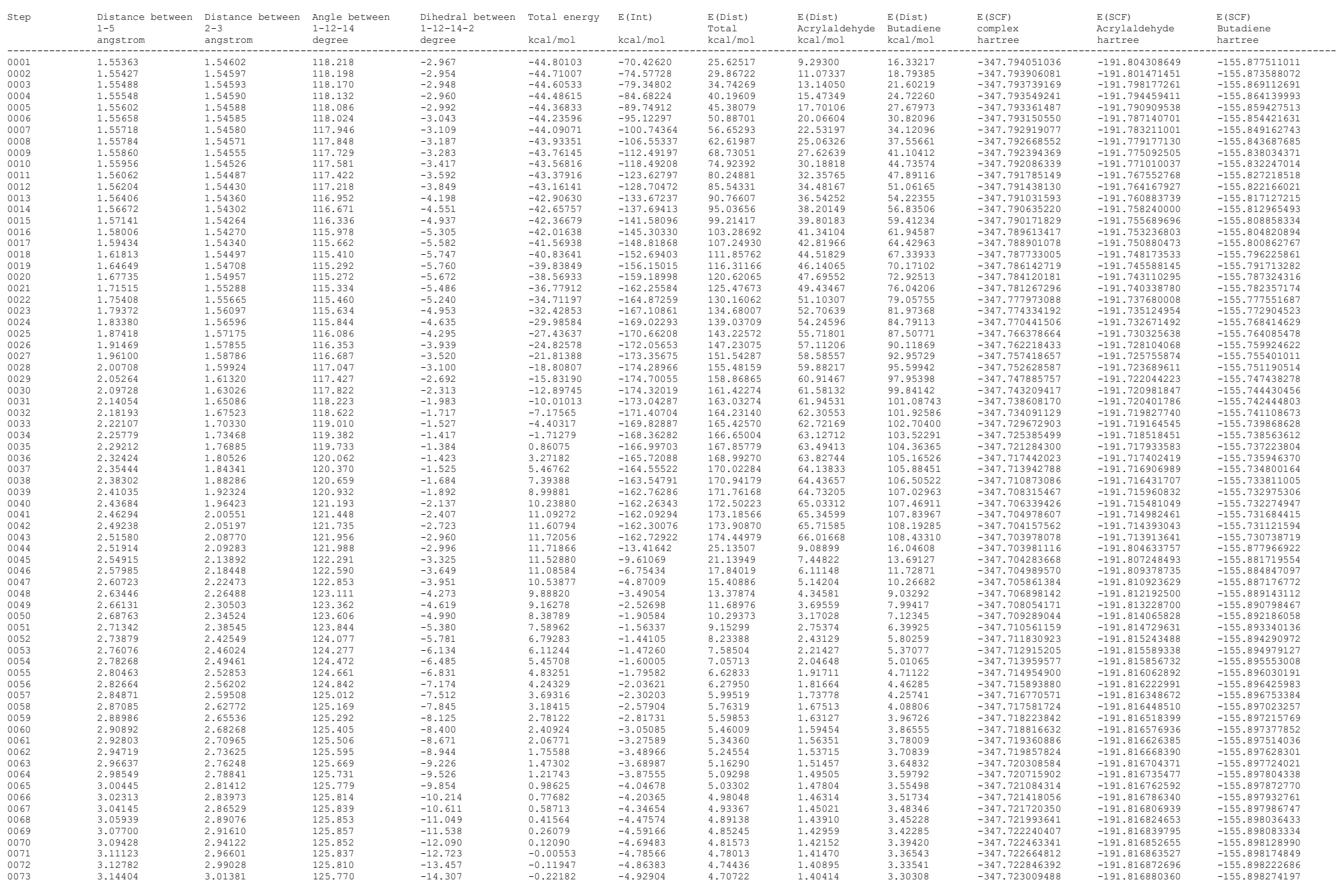




\section{Plotting}

Results of autoDIAS can be read by the numpy genfromtxt or the Excel data from text function. In the following a commented script of how to plot resulting data using Python 3.7.1 and Matplotlib are presented.

\section{Plotting of total, distortion and interaction energies for a single geometric parameter (used for Figure 5)}

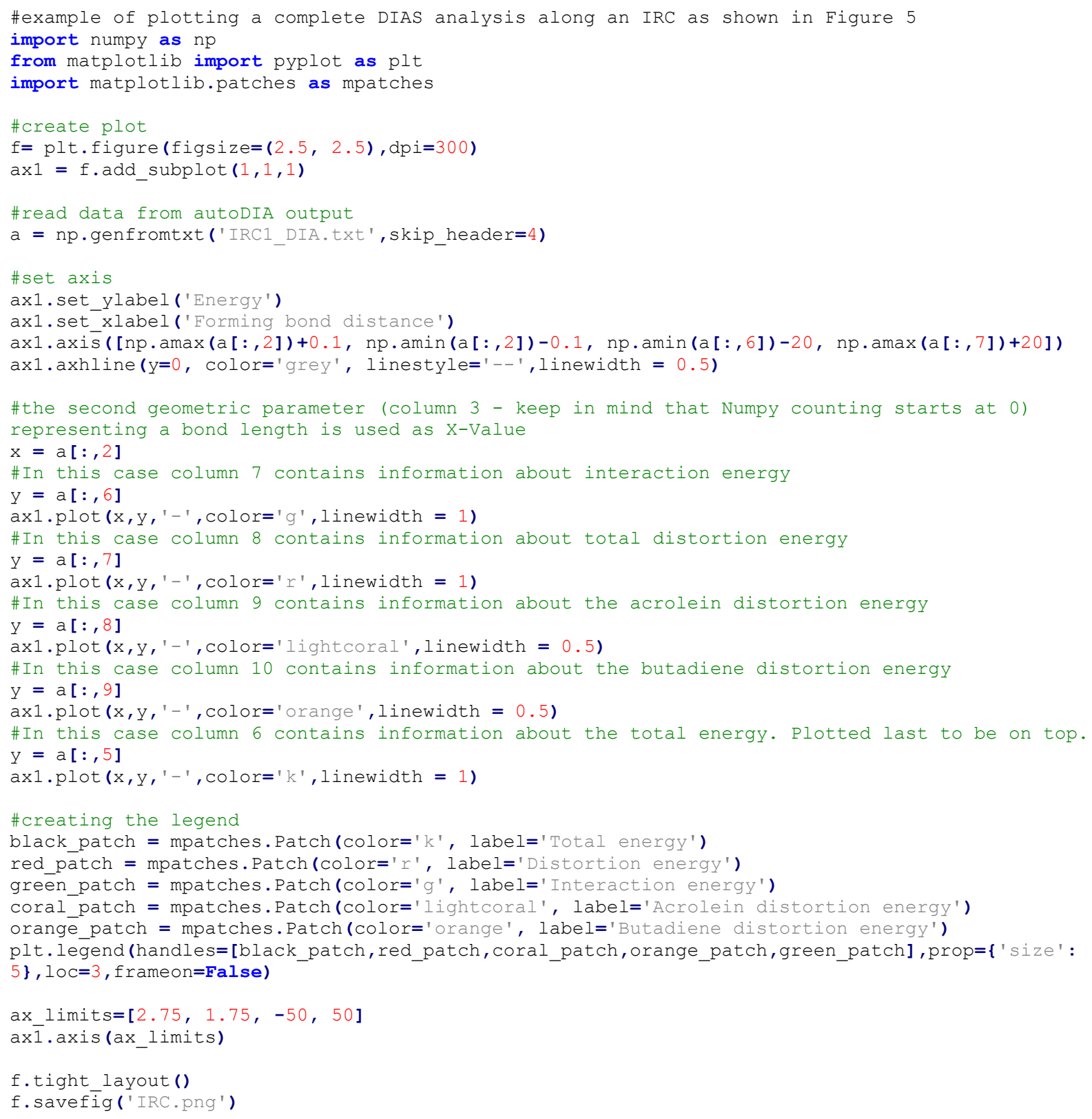


Other files

autoDIAS-1.0.1.zip (22.28 KiB)

view on ChemRxiv - download file 